\title{
MEASURING THE ROTATIONAL PERIODS OF ISOLATED MAGNETIC WHITE DWARFS
}

\author{
Carolyn S. Brinkworth ${ }^{1,2}$, Matthew R. Burleigh ${ }^{3}$, Katherine Lawrie $^{3}$, Thomas R. Marsh ${ }^{4}$, and Christian KnigGe ${ }^{5}$ \\ ${ }^{1}$ Spitzer Science Center, California Institute of Technology, Pasadena, CA 91125, USA \\ ${ }^{2}$ NASA Exoplanet Science Institute, California Institute of Technology, Pasadena, CA 91125, USA \\ ${ }^{3}$ Department of Physics and Astronomy, University of Leicester, Leicester LE1 7RH, UK \\ ${ }^{4}$ Department of Physics and Astronomy, University of Warwick, Coventry CV4 7AL, UK \\ ${ }^{5}$ School of Physics and Astronomy, University of Southampton, Southampton SO17 1BJ, UK \\ Received 2012 March 27; accepted 2013 June 22; published 2013 July 24
}

\begin{abstract}
We present time-series photometry of 30 isolated magnetic white dwarfs, surveyed with the Jacobus Kapteyn Telescope between 2002 August and 2003 May. We find that 9 were untestable due to varying comparison stars, but of the remaining $21,5(24 \%)$ are variable with reliably derived periods, while a further $9(43 \%)$ are seen to vary during our study, but we were unable to derive the period. We interpret the variability to be the result of rotation of the objects. We find no correlation between rotation period and mass, temperature, magnetic field, or age. We have found variability in 9 targets with low magnetic field strengths and temperatures low enough for partially convective atmospheres, which we highlight as candidates for polarimetry to search for starspots. Most interestingly, we have found variability in one target, PG1658+441, which has a fully radiative atmosphere in which conventional starspots cannot form, but a magnetic field strength that is too low to cause magnetic dichroism. The source of variability in this target remains a mystery.
\end{abstract}

Key words: stars: magnetic field - stars: rotation - starspots - surveys - white dwarfs

Online-only material: color figures

\section{INTRODUCTION}

Up to approximately 600 isolated white dwarfs, composing $\sim 3 \%$ of the currently known population, have measured magnetic fields $(10 \mathrm{kG}<B<1000 \mathrm{MG}$; e.g., Wickramasinghe \& Ferrario 2000; Schmidt et al. 2003; Vanlandingham et al. 2005; Kawka et al. 2007; Kepler et al. 2013; Kleinman et al. 2013). Holberg et al. (2008) have cataloged 16 magnetic degenerates among 126 white dwarfs within $20 \mathrm{pc}$, suggesting the fraction among the total population is at least $13 \%$. Kawka et al. (2007) list 9 magnetic stars from 43 white dwarfs in the more statistically complete sample within $13 \mathrm{pc}$, resulting in an incidence of $21 \% \pm 8 \%$. Furthermore, Jordan et al. (2007) have searched for magnetic fields as low as $1 \mathrm{kG}$ in a number of nearby white dwarfs, and estimate the fraction with such weak fields as $11 \%-15 \%$, similar to the incidence of higher field strengths.

The mass distribution of magnetic white dwarfs implies that they have a higher average mass than their non-magnetic counterparts (e.g., Liebert et al. 2005). This bias may provide a clue as to the origin of their magnetic fields. Some of these stars may be the direct descendants of high-mass main-sequence magnetic Ap and Bp stars $\left(M>2 M_{\odot}\right.$; Wickramasinghe \& Ferrario 2005). However, Kawka \& Vennes (2004) argued that the present space density of $\mathrm{Ap} / \mathrm{Bp}$ progenitors is insufficient to account for the density of known magnetic white dwarfs in the solar neighborhood.

Liebert et al. (2005) noted that although $\sim 25 \%$ of interacting cataclysmic variable $(\mathrm{CV})$ systems contain a magnetic white dwarf, there are no known examples of detached binaries consisting of a main-sequence star and a magnetic white dwarf. In other words, there are no known close binary progenitors of magnetic CVs. Tout et al. (2008) explain this discrepancy as the result of the common envelope evolution that precedes the formation of a detached close binary. During a common envelope, a low-mass star and the eventual white dwarf are brought close together as the orbit shrinks through friction and the loss of angular momentum. A CV later forms when the emergent close binary is brought into contact by magnetic braking or gravitational radiation. Tout et al. (2008) propose that the magnetic field on the white dwarf is generated by differential rotation and convection within the common envelope. The smaller the orbital separation at the end of the common envelope phase, the stronger the magnetic field on the white dwarf. Magnetic CVs are systems which emerge from the common envelope very close to semi-detached contact. The isolated, single magnetic white dwarfs are the result of common envelope binaries that merged. Thus, Tout et al. (2008) propose that all highly magnetic white dwarfs, whether single or in magnetic $\mathrm{CVs}$, are the result of close binary evolution. This scenario of forming high-field magnetic white dwarfs through mergers is supported by the more recent work of Nordhaus et al. (2011) and Garcia-Berro et al. (2012). In this picture, low-field stars $(<\mathrm{a} M G)$ may still be the descendants of single main-sequence progenitors.

Measuring rotational periods in non-magnetic white dwarfs is notoriously difficult due to the heavy broadening of the spectral lines by the strong gravitational field (Berger et al. 2005). Magnetic white dwarfs, on the other hand, display spectroscopic and/ or photometric variability which allows much easier identification of their spin periods. The rotation rates allow investigation of angular momentum transfer from the core to the envelope and large-scale angular momentum loss during post-main-sequence evolution, for both magnetic white dwarfs and white dwarfs in general, and for investigation of the formation scenarios.

Spectroscopic variability in magnetic white dwarfs is generally caused by variations in the surface field strength, which can be observed in the motion of the Zeeman-split components of the Balmer lines. Photometric variability can be caused by the dependence of the continuum opacity on the surface field 


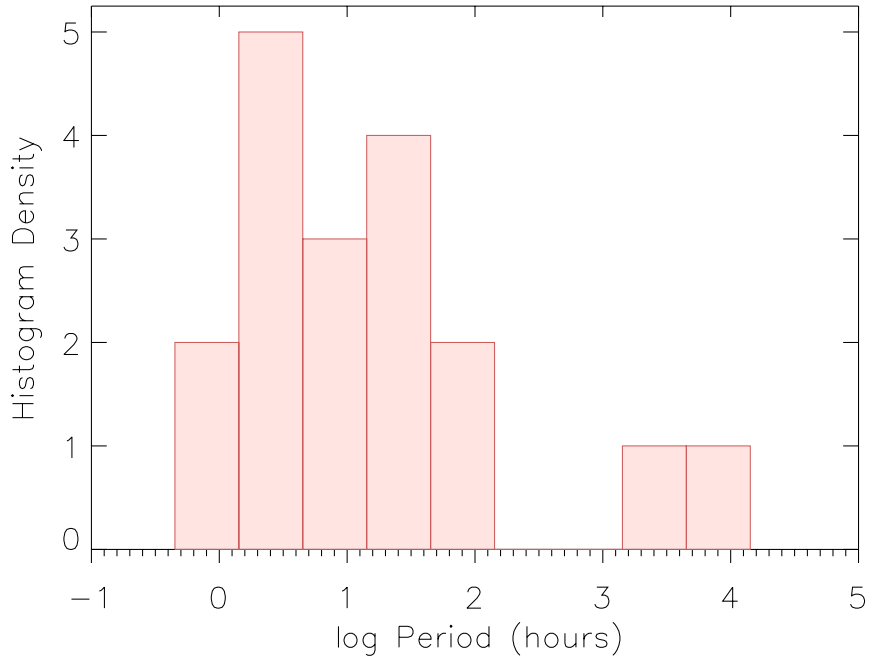

Figure 1. Histogram showing the period distribution of our targets with observed variability. We also include the white dwarfs WD1953-011 and GD356 from Brinkworth et al. (2004, 2005).

(A color version of this figure is available in the online journal.)

strength, called magnetic dichroism (Ferrario et al. 1997), but this requires a very high magnetic field strength. For example, the white dwarf REJ0317-349 shows optical photometric modulations $>10 \%$ peak-to-peak (Barstow et al. 1995) due to the effects of magnetic dichroism in a field which varies from 180 to $800 \mathrm{MG}$ across the stellar surface (Ferrario et al. 1997; Burleigh et al. 1999; Vennes et al. 2003).

Variability in lower field strength magnetic white dwarfs may be due to starspots in a convective atmosphere. Starspots are caused by the inhibition of convection in the stellar atmosphere by the magnetic field. DA white dwarfs become fully radiative above temperatures of 12,000-14,000 K, and DB white dwarfs around 23,000-28,000 K, so magnetic white dwarfs below that temperature should be capable of forming starspots. These areas of reduced convection are cooler (and therefore darker) than the surrounding atmosphere, leading to a drop in the observed magnitude of the star as the spots rotate into view. The effect is evident in the $T_{\text {eff }} \approx 8000 \mathrm{~K}, B \approx 70 \mathrm{kG}$ star WD1953-011, which shows a sinusoidal variation of $\approx 2 \%$ peak-to-peak amplitude every 1.44 days due to a starspot covering $\sim 10 \%$ of the surface (Maxted et al. 2000; Brinkworth et al. 2005; Valyavin et al. 2011).

Previously measured magnetic white dwarf rotational periods show evidence for a bimodal distribution, with one group rotating very slowly, possibly with periods of $>100 \mathrm{yr}$, and another group rotating more quickly, of order minutes-hours (see Figure 1). Spruit (1998) proposed that the extremely slow rotators could be produced if the magnetic field locks the forming white dwarf to the escaping envelope of its progenitor, very efficiently shedding angular momentum, while King et al. (2001) have suggested that the fastest rotators ( $P_{\text {rot }} \sim$ minutes) may have been spun up in double-degenerate mergers. Non-magnetic white dwarfs, meanwhile, have periods measured by either line broadening or the splitting of modes seen in the Fourier transform analysis (Kepler et al. 1995), of approximately a day (Koester et al. 1998; Kawaler 2004; Karl et al. 2005; Berger et al. 2005). In comparison, the longest period non-radial pulsator yet discovered has a period of $4000 \mathrm{~s}$ (Hermes et al. 2012), but this object has an unusually low mass $\left(0.17 M_{\odot}\right)$ and surface gravity $(\log g \sim 6)$. Most pulsating white dwarfs have surface gravities of around $\log g \sim 8$, similar to most of the targets in our sample, and have pulsation periods of around 100-1000 s (Winget \& Kepler 2008; Mukadam et al. 2006). Variability on timescales of more than about 20 minutes can therefore more likely be attributed to rotation rather than pulsations, unless the target has a particularly low mass.

In order to further investigate the rotation periods of magnetic white dwarfs and to compare them to white dwarfs in general, we have conducted a survey of 30 single, northern hemisphere magnetic white dwarfs $V<17$ for photometric variability. The discovery of periodic variability in the cool stars GD356 $\left(T_{\mathrm{eff}}=\right.$ $7500 \mathrm{~K})$ and WD1953-011 has been reported previously (Brinkworth et al. 2004, 2005). Here, we report in detail results for the remainder of the sample, and search for correlations between rotation rate and other intrinsic parameters (e.g., temperature, mass, age) to aid investigation of the formation scenarios for magnetic white dwarfs, and for comparison and contrasting with the determination of rotational velocities in non-magnetic degenerates. A full list of the targets and their parameters as gleaned from the literature can be found in Table 1.

\section{OBSERVATIONS AND DATA REDUCTION}

The data were collected over three individual weeks of time on the $1.0 \mathrm{~m}$ Jacobus Kapteyn Telescope, which was part of the Isaac Newton Group of telescopes at the Observatorio del Roque de los Muchachos of the Instituto de Astrofisica de Canarias. The observations were all taken in the Harris $V$ band, using the SITe1 CCD chip $(2088 \times 2120$ pixels, readout noise $=6 e$, gain $=$ $1.9 e \mathrm{ADU}^{-1}$, pixel size $=15 \mu \mathrm{m}$, image scale $=0.33$ pixel $^{-1}$ ), during 2002 August, 2003 February, and 2003 May. In total, we observed 33 targets from the list of isolated magnetic white dwarfs contained in Wickramasinghe \& Ferrario (2000). Of these targets, we were able to observe 14 over more than one week to search for variability over timescales of weeks-months. A full list of observations is included in Table 2.

The data were reduced using the ULTRACAM pipeline software (Dhillon et al. 2007). Bias frames for each night were combined to create a master bias, which was subtracted from all of the data frames. Sky flats were checked, and any with counts less than 10,000 or greater than 35,000 were discarded. The remaining flat fields were combined to create a master flat for each night, before normalizing the master and dividing it through the bias-corrected data frames. There were no sky flats for 2003 February 22, 25, or 26, so the data frames from those nights were flat-fielded using combined, normalized dome flats.

Differential photometry was performed on the targets with respect to at least two bright comparison stars in the field, and usually three or four. We used variable apertures (allowing the apertures to vary with the fitted FWHM of the source, so compensating for changes in the observing conditions). We extracted fluxes by summing counts in excess of the sky level, which was estimated by taking the mean after rejecting points more than $3 \sigma$ away from the mean.

The stable comparisons were combined to create a single bright master comparison, which we divided into the target to calculate the differential photometry. Results are given in differential flux and all times have been converted to Heliocentric Julian Date.

\subsection{Detection of Variability}

We used a "floating mean" periodogram (e.g., Cumming et al. 1999; Morales-Rueda et al. 2003) to search for periodicity in 
Table 1

Magnetic White Dwarf Parameters from the Literature

\begin{tabular}{|c|c|c|c|c|c|c|c|c|c|}
\hline Target & White Dwarf Name & $\begin{array}{c}B_{p} \\
(\mathrm{MG})\end{array}$ & $\begin{array}{l}T_{\text {eff }} \\
(\mathrm{K})\end{array}$ & $\begin{array}{l}\text { Mass } \\
\left(M_{\odot}\right)\end{array}$ & Comp & $\begin{array}{c}V \\
(\mathrm{mag})\end{array}$ & $\begin{array}{l}\text { Age } \\
(\mathrm{Gyr})\end{array}$ & $P_{\text {lit }}$ & Refs \\
\hline EUVE J1439+75.0 & WD1440+753 & 14.8 & 39500 & $0.9-1.2$ & $\mathrm{H} ; \mathrm{DD}$ & 15.4 & $0.005-0.3$ & $\ldots$ & 44,9 \\
\hline G99-37 & WD0548-001 & 10-20: & 6070 & 0.69 & $\mathrm{He}$ & 14.6 & 3.90 & $4.117 \mathrm{hr}$ & $1,9,50,51$ \\
\hline G99-47 & WD0533+053 & 20 & 5790 & 0.71 & $\mathrm{H}$ & 14.1 & 3.97 & $1 \mathrm{hr}$ & $1,9,10,11,12,55$ \\
\hline G111-49 & WD0756+437 & $300-377$ & 8500 & 1.07 & $\mathrm{H}$ & 16.3 & $\ldots$ & $\ldots$ & $3,9,15,45$ \\
\hline G141-2 & WD1818+126 & 3: & 6340 & 0.26 & $\mathrm{H} ; \mathrm{DD}$ & 15.9 & 0.93 & years? & $1,34,22$ \\
\hline G158-45 & WD0011-134 & 16.7 & 6010 & 0.71 & $\mathrm{H}$ & 15.9 & 3.43 & $11 \mathrm{hr}-1$ day & 1,3 \\
\hline G183-35 & WD1814+248 & 14: & 6500 & $\ldots$ & $\mathrm{H}, \mathrm{He}:$ & 16.9 & $\ldots$ & 50 minutes-few yr & 3,28 \\
\hline G195-19 & WD0912+536 & 100 & 7160 & 0.75 & $\mathrm{He}$ & 13.8 & 2.54 & 1.33 days & $1,17,18$ \\
\hline $\mathrm{G} 217-037$ & WD0009+501 & $\simeq 0.2$ & 6540 & 0.74 & $\mathrm{H}$ & 14.4 & 3.58 & $2-20 \mathrm{hr}$ & $1,2,48,55$ \\
\hline G227-28 & WD1820+609 & $\leqslant 0.1$ & 4780 & 0.48 & $\mathrm{H}$ & 15.7 & 4.68 & $\ldots$ & $1,3,55$ \\
\hline G227-35 & WD1829+547 & $170-180$ & 6280 & 0.90 & $\mathrm{H}$ & 15.5 & 4.76 & $\geqslant 100 \mathrm{yr}$ & $12,1,35$ \\
\hline $\mathrm{G} 234-4$ & WD0728+642 & $0.1-0.4$ & 4500 & 0.58 & $\mathrm{H}, \mathrm{He}$ & 16.3 & 7.58 & $\ldots$ & 3,55 \\
\hline G240-72 & WD1748+708 & $\geqslant 100$ & 5590 & 0.81 & $\mathrm{He}$ & 14.2 & 5.69 & $\geqslant 100$ yr: & $1,33,17,55$ \\
\hline G256-7 & WD1309+853 & 4.9 & 5600 & $\ldots$ & $\mathrm{H}$ & 16.0 & $\ldots$ & $\ldots$ & 28 \\
\hline GD77 & WD0637+478 & 1.2 & 14000 & $0.74:$ & $\mathrm{H}$ & 14.8 & $\ldots$ & $\ldots$ & $9,13,14$ \\
\hline GD90 & WD0816+376 & 9 & 11000 & 0.60 & $\mathrm{H}$ & 15.6 & 0.47 & $\ldots$ & $3,14,16,52,53$ \\
\hline GD229 & WD2010+310 & 500 & 16000 & $\geqslant 1.0$ & $\mathrm{He}$ & 14.8 & $\ldots$ & $\geqslant 100 \mathrm{yr}$ & $37,38,33,39$ \\
\hline Grw $+70^{\circ} 8247$ & WD1900+705 & 320 & 12070 & 0.95 & $\mathrm{He}$ & 13.2 & 0.95 & $\geqslant 100 \mathrm{yr}$ & $1,36,20,55$ \\
\hline HE0107-0158 & WD0107-019 & 10:-30: & $\ldots$ & $\ldots$ & $\mathrm{He}$; Bin & 16.4: & $\ldots$ & $\ldots$ & 4,5 \\
\hline HE1045-0908 & WD1045-091 & $10-75$ & 10000 & $\ldots$ & $\mathrm{H}$ & 16.7 & $\ldots$ & $2-11 \mathrm{hr}$ & $5,25,54$ \\
\hline HE1211-1707 & WD1211-171 & 50 & $12-23000$ & $\ldots$ & $\mathrm{He}$ & 16.8 & $\ldots$ & $\sim 2 \mathrm{hr}$ & 5,26 \\
\hline LB8915 & WD0853+163 & $0.1-1$ & $21-27000$ & $\ldots$ & $\mathrm{H}+\mathrm{He}$ & 15.8 & $\ldots$ & $\ldots$ & 3,49 \\
\hline LB11146 & WD0945+246 & 670 & 16000 & 0.9 & H, He; DD & 14.3 & $\ldots$ & $\ldots$ & $41,42,43$ \\
\hline LHS2273 & WD1026+117 & $\sim 10$ & 7160 & 0.59 & $\mathrm{H}$ & 16.6 & 1.51 & $\ldots$ & 22,23 \\
\hline LHS5064 & WD $0257+080$ & $\leqslant 0.2$ & 6680 & 0.57 & $\mathrm{H}$ & 15.9 & 1.60 & $\ldots$ & 1,9 \\
\hline LP907-037 & WD1350+090 & $\leqslant 0.1$ & 9520 & 0.83 & $\mathrm{H}$ & 14.6 & 1.32 & $\ldots$ & $30,2,47$ \\
\hline PG0136+251 & WD0136+251 & $\leqslant 0.1$ & 39640 & 1.20 & $\mathrm{H}$ & 15.8: & 0.75 & $\ldots$ & 6,47 \\
\hline PG1015+015 & WD1015+014 & $50-90$ & 10000 & $\ldots$ & $\mathrm{H}$ & 16.3 & $\ldots$ & 98.7 minutes & $19,20,21,46$ \\
\hline PG1031+234 & WD1031+234 & $500-1000$ & 15000 & 0.93 & $\mathrm{H}$ & 15.8 & 0.55 & $3.40 \mathrm{hr}$ & 24,47 \\
\hline PG1533-057 & WD1533-057 & 31 & 20000 & 0.94 & $\mathrm{H}$ & 15.3 & 0.25 & $\sim 1$ day & $31,23,47$ \\
\hline PG1658+441 & WD1658+441 & 3.5 & 30510 & 1.31 & $\mathrm{H}$ & 14.6 & 0.38 & $\ldots$ & $32,6,47$ \\
\hline PG2329+267 & WD2329+267 & 2.3 & 11730 & 1.18 & $\mathrm{H}$ & 15.3 & 2.04 & $\ldots$ & 40,47 \\
\hline SBSS1349+545 & WD1349+545 & 760 & 11000: & $\ldots$ & $\mathrm{H}$ & 16.4 & $\ldots$ & $\ldots$ & 29 \\
\hline
\end{tabular}

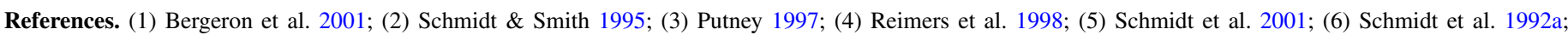

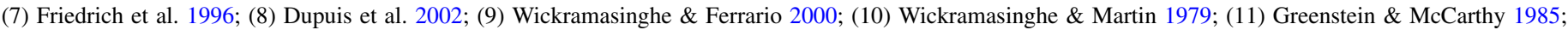

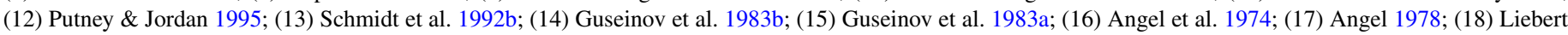

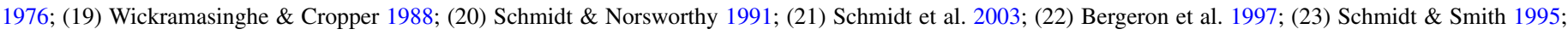

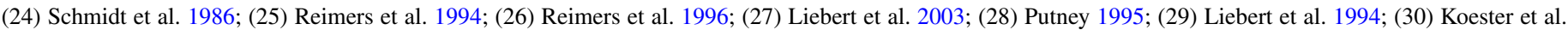

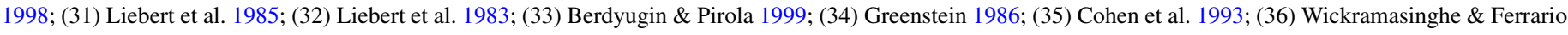

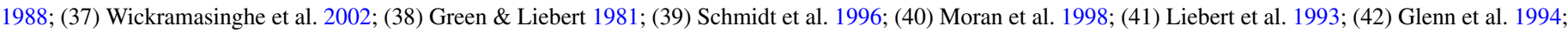

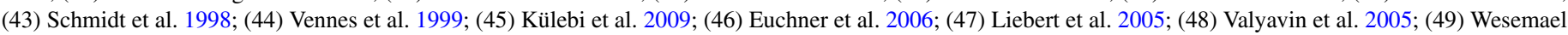

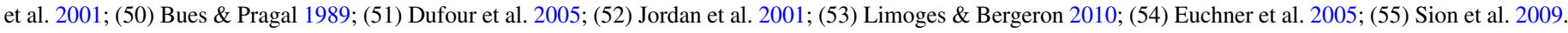

each of the targets. This is a generalization of the Lomb-Scargle periodogram (Lomb 1976; Scargle 1982) and involves fitting the data with a sinusoid plus constant of the form

$$
A+B \sin \left[2 \pi f\left(t-t_{0}\right)\right]
$$

where $f$ is the frequency and $t$ is the observation time. We searched frequencies from 0.001 to 100 cycles day ${ }^{-1}$, with an oversampling factor of 10 . For the normalization of the periodogram, we use the weighted sum of squares of residuals to the best-fit sinusoid, $\chi^{2}\left(\omega_{0}\right)$, as described in Cumming et al. (1999). The advantage of the floating mean periodogram over the Lomb-Scargle periodogram is that it treats the constant, $A$, as an extra free parameter rather than fixing the zero-point and then fitting a sinusoid, i.e., it allows the zero-point to "float" during the fit. It also takes uncertainties into account, while the Lomb-Scargle periodogram ignores them. Unlike the
Lomb-Scargle periodogram and running Fourier transforms, the floating-mean periodogram is robust when the number of observations is small, the sampling is uneven, and the derived period is longer than the baseline of the observations (Cumming et al. 1999). The resultant periodogram is an inverted $\chi^{2}$ plot of the fit at each frequency.

To test the significance of the variability, we carried out two further tests. Firstly, we suspected that the formal errors were underestimated, so we re-scaled the error bars to give a reduced $\chi^{2}=1$ for the global minimum in the periodogram. We then compared the $\chi^{2}$ values from the periodogram to the $\chi^{2}$ of a constant fit, using a change in $\chi^{2}$ of 16 (equivalent to $4 \sigma$ ) as the threshold for a target to be initially classified as variable. A list of the reduced $\chi^{2}$ values for all of the targets can be found in Table 3. Having established that the formal errors failed to cover the scatter in the data, we compared the level of variability in the target to the level seen in the comparison stars. We compared the 
Table 2

Observing Log for Our White Dwarf Sample

\begin{tabular}{|c|c|c|c|}
\hline Target & White Dwarf Name & Epoch & Number of Frames \\
\hline G217-037 & WD0009+501 & $2002 \mathrm{Aug}$ & 198 \\
\hline G158-45 & WD0011-134 & 2002 Aug & 76 \\
\hline HE0107-0158 & WD0107-019 & $2002 \mathrm{Aug}$ & 60 \\
\hline PG0136+251 & WD0136+251 & 2002 Aug & 25 \\
\hline LHS5064 & WD 0257+080 & $2003 \mathrm{Feb}$ & 33 \\
\hline G99-47 & WD0533+053 & $2003 \mathrm{Feb}$ & 69 \\
\hline G99-37 & WD0548-001 & $2003 \mathrm{Feb}$ & 23 \\
\hline GD77 & WD0637+478 & $2003 \mathrm{Feb}$ & 45 \\
\hline G234-4 & WD0728+642 & $2003 \mathrm{Feb}$ & 18 \\
\hline G111-49 & WD0756+437 & $2003 \mathrm{Feb}$ & 100 \\
\hline GD90 & WD0816+376 & $2003 \mathrm{Feb}$ & 18 \\
\hline LB8915 & WD0853+163 & $2003 \mathrm{Feb}$ & 30 \\
\hline \multirow[t]{2}{*}{ G195-19 } & WD0912+536 & $2003 \mathrm{Feb}$ & 33 \\
\hline & & 2003 May & 40 \\
\hline LB11146 & WD0945+246 & $2003 \mathrm{Feb}$ & 18 \\
\hline PG1015+015 & WD1015+014 & 2003 Feb & 26 \\
\hline LHS2273 & WD1026+117 & 2003 May & 35 \\
\hline PG1031+234 & WD1031+234 & $2003 \mathrm{Feb}$ & 25 \\
\hline HE1045-0908 & WD1045-091 & $2003 \mathrm{Feb}$ & 47 \\
\hline \multirow[t]{2}{*}{ HE1211-1707 } & WD1211-171 & $2003 \mathrm{Feb}$ & 21 \\
\hline & & 2003 May & 22 \\
\hline G256-7 & WD1309+853 & 2003 May & 37 \\
\hline SBSS1349+545 & WD1349+545 & 2003 May & 51 \\
\hline LP907-037 & WD1350+090 & 2003 May & 30 \\
\hline \multirow[t]{3}{*}{ EUVE J1439+75.0 } & WD1440+753 & 2002 Aug & 20 \\
\hline & & $2003 \mathrm{Feb}$ & 13 \\
\hline & & 2003 May & 5 \\
\hline \multirow[t]{3}{*}{ PG1533-057 } & WD1533-057 & 2002 Aug & 68 \\
\hline & & $2003 \mathrm{Feb}$ & 21 \\
\hline & & 2003 May & 306 \\
\hline \multirow[t]{2}{*}{ PG1658+441 } & WD1658+441 & 2002 Aug & 55 \\
\hline & & 2003 May & 10 \\
\hline \multirow[t]{2}{*}{$\mathrm{G} 240-72$} & WD1748+708 & 2002 Aug & 60 \\
\hline & & 2003 May & 10 \\
\hline \multirow[t]{2}{*}{ G183-35 } & WD1814+248 & $2002 \mathrm{Aug}$ & 78 \\
\hline & & 2003 May & 40 \\
\hline G141-2 & WD1818+126 & 2002 Aug & 66 \\
\hline \multirow[t]{2}{*}{ G227-28 } & WD1820+609 & 2002 Aug & 40 \\
\hline & & 2003 May & 10 \\
\hline \multirow[t]{2}{*}{$\mathrm{G} 227-35$} & WD1829+547 & 2002 Aug & 55 \\
\hline & & 2003 May & 10 \\
\hline Grw $+70^{\circ} 8247$ & WD1900+705 & 2002 Aug & 125 \\
\hline \multirow[t]{2}{*}{ GD229 } & WD2010+310 & 2002 Aug & 55 \\
\hline & & 2003 May & 10 \\
\hline \multirow[t]{2}{*}{ PG2329+267 } & WD2329+267 & 2002 Aug & 153 \\
\hline & & 2003 May & 5 \\
\hline
\end{tabular}

Note.Targets are listed in order of R.A.

rms of the normalized target differential light curve to the rms of the normalized differential light curves of the comparisons. For those with target rms comparable to the comparison rms, we ran a periodogram on the comparison stars to test for periodicity in the comparison star data. Those targets that display variability on the same level as the comparisons, with similar significance in the periodogram compared to constant fits, are listed as non-variable. Finally, we ran Monte Carlo simulations with Gaussian noise to estimate the false alarm probability (FAP) for each target, as outlined in detail in Cumming et al. (1999). The results of the FAP tests can be found in Table 3. The FAP was calculated as the fraction of 1000 randomly generated datasets (sampled at the observed times about the observed mean flux with random Gaussian noise) that would cause a mean power signal in the periodogram greater than the mean power found from our observed data. We set the significance threshold to an FAP of $1 \%($ FAP $<0.01)$.

The final classification of variable versus non-variable was made using a combination of the above methods. The identification of the best-fitting period for the variable targets was made by examining the change in the $\chi^{2}$ between the global minimum and the next best frequency. Lines marking changes in $\chi^{2}$ of 1,4 , and 9 (equivalent to $1 \sigma, 2 \sigma$, and $3 \sigma$, assuming one fitted parameter) are marked on the periodograms for each target. The $2 \sigma$ errors are quoted in the text, and a change in $\chi^{2}$ of $9(3 \sigma)$ between two minima was taken as a significantly better fit.

For some of the targets (e.g., LB8915) there were a number of local aliases within the $3 \sigma$ uncertainty. In these cases, we ran a Lomb-Scargle periodogram on the data, which ignores the error bars on the data, giving us an unweighted least-squares fitting as an alternative test. We then folded the light curves on at least the best two periods identified in the two periodograms. The most likely periods for each of the targets are quoted in Table 3, along with the $2 \sigma$ uncertainties.

\section{RESULTS}

Of the 33 stars we observed, two were identified in the literature as binary systems, and one is not believed to be magnetic. These stars were discarded from our survey, leaving us with 30 isolated magnetic white dwarfs. Of these 30 targets, 5 displayed photometric variability with a measurable period, i.e., a minimum in the periodogram that was significantly better than the next-best-fitting period. A further 9 displayed significant variability, but we were unable to differentiate between a number of possible aliases. We found no evidence for variability in the remaining targets, but provide upper limits.

\subsection{Targets Displaying Variability with a Measured Period}

$$
\text { 3.1.1. G111-49 (WD0756+437) }
$$

The magnetic nature of G111-49 was discovered by Putney (1995), who reclassified its spectral type as an H-rich magnetic white dwarf and reported strong circular polarization $\left(V_{\max }=\right.$ $9 \%$ ) and a field strength of $\sim 220$ MG. Putney (1995) also noted that G111-49 has been classified in the literature as a variety of different white dwarf spectral types since Greenstein et al. (1977) and suggested that this could be indicative of rotation. More recently, Külebi et al. (2009) measured the field strength from a Sloan optical spectrum as $300 \mathrm{MG}$ (centered magnetic dipole case) or $377 \mathrm{MG}$ if the dipole is shifted by 0.23 stellar radii along the dipole axis. We have discovered photometric variability in G111-49 with a peak-to-peak amplitude of $\sim 7 \%$ and a period of $6.68{ }_{-0.03}^{+0.02} \mathrm{hr}$. The periodogram is in Figure 2, which shows that the difference in $\chi^{2}$ between the fits of the best and second-best periods $\left(\Delta \chi^{2}\right)$ is more than 200 . The light curve, folded on the best period, is also shown in Figure 2.

$$
\text { 3.1.2. HE1211-1707 (WD1211-171) }
$$

Reimers et al. (1996) reported time-variable absorption features in the spectrum of HE1211-1707, and Schmidt et al. (2001) found the rotational period from optical spectra and circular spectropolarimetry to be $\sim 2 \mathrm{hr}$. Schmidt et al. (2001) and Jordan et al. (2001) also independently showed that HE1211-1707 is a rare He-rich magnetic degenerate. We observed HE1211-1707 in 2003 February, but failed to find any 

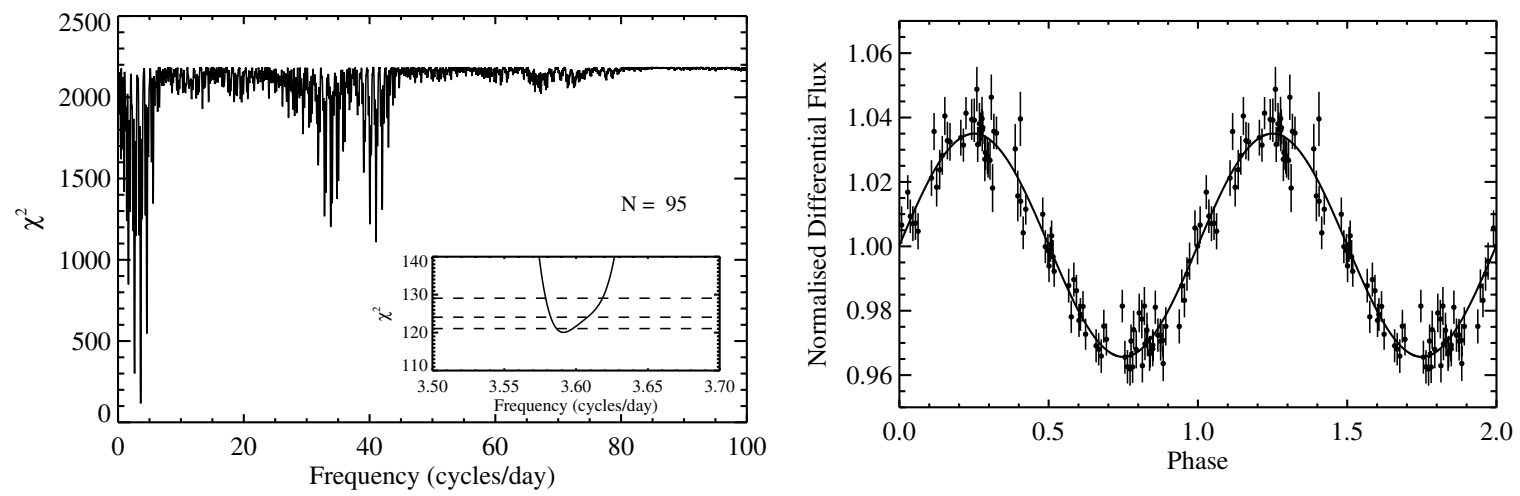

Figure 2. Left: periodogram for G111-49. Inset: periodogram zoomed in on the best-fitting period of $6.68 \mathrm{hr}$. Dashed lines show the $1 \sigma, 2 \sigma$, and $3 \sigma$ uncertainty levels. Right: light curve for G111-49, folded on the best-fitting period of $6.68 \mathrm{hr}$. Fitting parameters can be found in Table 3 .
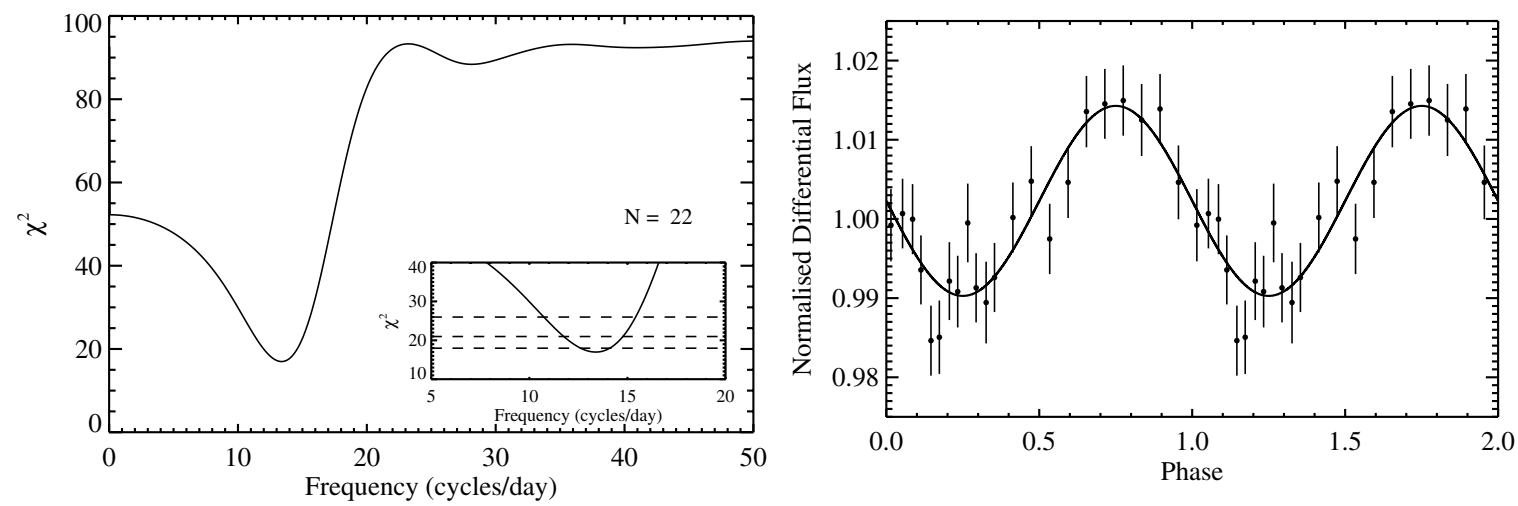

Figure 3. Left: periodogram for HE1211-1707. Inset: periodogram zoomed in on the best-fitting period of $1.79 \mathrm{hr}$. Dashed lines show the $1 \sigma, 2 \sigma$, and $3 \sigma$ uncertainty levels. Right: light curve for HE1211-1707, folded on the best-fitting period of $1.79 \mathrm{hr}$. Fitting parameters can be found in Table 3 .

Table 3

The Top Two Sections Show the Period Fits for the Variable Magnetic White Dwarfs

\begin{tabular}{|c|c|c|c|c|c|c|}
\hline Target & White Dwarf Name & $\begin{array}{l}\text { Period from } \\
\text { Literature }\end{array}$ & $\begin{array}{l}\text { Calculated } \\
\text { Period }\end{array}$ & $\begin{array}{c}\text { Reduced } \\
\chi^{2}\end{array}$ & $\begin{array}{c}\text { Semi-amplitude } \\
(\%)\end{array}$ & $\begin{array}{l}\text { Variable } \\
\text { FAP }\end{array}$ \\
\hline G111-49 & WD0756+437 & $\ldots$ & $6.68_{-0.03}^{+0.02} \mathrm{hr}$ & 1.31 & 3.5 & $<0.001$ \\
\hline HE1211-1707 & WD1211-171 & $\sim 2 \mathrm{hr}$ & $1.79_{-0.16}^{+0.25} \mathrm{hr}$ & 0.89 & 1.2 & $<0.001$ \\
\hline PG1015+015 & WD1015+014 & 98.7 minutes & $105_{-8}^{+12}$ minutes & 1.4 & 2.3 & $<0.001$ \\
\hline PG1031+234 & WD1031+234 & $3.40 \mathrm{hr}$ & $3.53 \pm 0.05 \mathrm{hr}$ & 1.3 & 6.9 & $<0.001$ \\
\hline$\underline{\text { PG1533-057 }}$ & WD1533-057 & $\sim 1$ day & $1.890 \pm 0.001 \mathrm{hr}$ & 2.1 & 0.5 & $<0.001$ \\
\hline G99-47 & WD0533+053 & $1 \mathrm{hr}$ & $26.8 \pm 0.7$ minutes & 1.0 & 0.3 & 0.003 \\
\hline G217-037 & WD0009+501 & $2-20 ? \mathrm{hr}$ & $\mathrm{hr}-2.5$ days & $\ldots$ & $\sim 1.3$ & $<0.001$ \\
\hline G227-28 & WD1820+609 & $\ldots$ & months-years & $\ldots$ & $\geqslant 1.5$ & $<0.001$ \\
\hline G240-72 & WD1748+708 & $\geqslant 100 \mathrm{yr}:$ & months & $\ldots$ & $\geqslant 1.3$ & $<0.001$ \\
\hline LB8915 & WD0853+163 & $\ldots$ & $2-24 \mathrm{hr}$ & $\ldots$ & $\sim 2.2$ & $<0.001$ \\
\hline LHS2273 & WD1026+117 & $\ldots$ & $35-45$ minutes & $\ldots$ & $\sim 0.7$ & 0.073 \\
\hline LHS5064 & WD $0257+080$ & $\ldots$ & $9 \mathrm{hr}-6$ days & $\ldots$ & 2.2 & $<0.001$ \\
\hline PG1658+441 & WD1658+441 & $\ldots$ & $6 \mathrm{hr}-4$ days & $\ldots$ & $\sim 1.1$ & $<0.001$ \\
\hline PG2329+267 & WD2329+267 & $\ldots$ & $2.767 \mathrm{hr}$ & 3.1 & 0.7 & $<0.001$ \\
\hline G158-45 & WD0011-134 & $11 \mathrm{hr}-1$ day & 30 minutes-days & $\ldots$ & 0.05 & 0.785 \\
\hline G183-35 & WD1814+248 & $\ldots$ & $\ldots$ & $\ldots$ & 2 & 0.344 \\
\hline GD90 & WD0816+376 & $\ldots$ & $\ldots$ & $\ldots$ & 2 & 0.827 \\
\hline Grw $+70^{\circ} 8247$ & WD1900+70 & $\ldots$ & $\ldots$ & $\ldots$ & 2 & 0.002 \\
\hline HE1045-0908 & WD1045-0908 & $\ldots$ & $\ldots$ & $\ldots$ & 1 & 0.139 \\
\hline LB11146 & WD0945+246 & $\ldots$ & $\ldots$ & $\ldots$ & $\ldots$ & 0.011 \\
\hline
\end{tabular}

Notes. The bottom section lists the isolated magnetic white dwarfs without significant variability, and quotes the upper limits that we were able to place on variability in these targets. The binary systems, non-magnetic system, and targets with variable or insufficient comparison stars have not been included. 

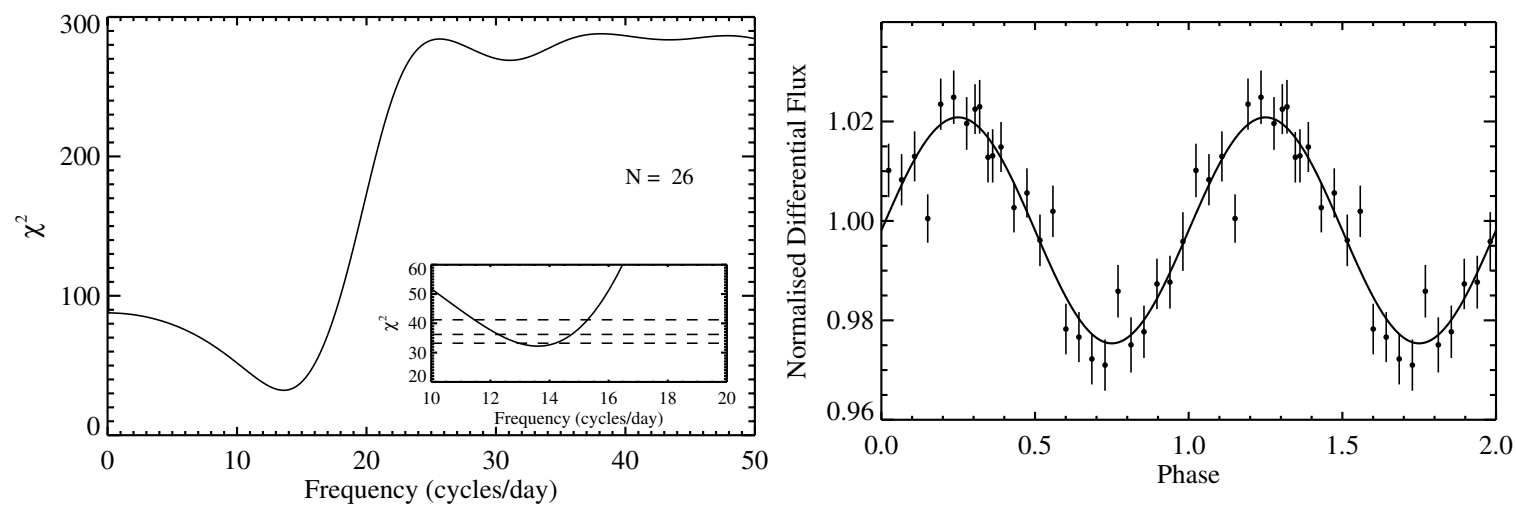

Figure 4. Left: periodogram for PG1015+015. Inset: periodogram zoomed in on the best-fitting period of 105 minutes. Dashed lines show the $1 \sigma, 2 \sigma$, and $3 \sigma$ uncertainty levels. Our measured frequency of 13.7 cycles day $^{-1}$ is within $2 \sigma$ of the previously reported value of 14.6 cycles day $^{-1}$ (Wickramasinghe \& Cropper 1988 ). Right: light curve for PG1015+015, folded on the best-fitting period of 105 minutes. Fitting parameters can be found in Table 3 .
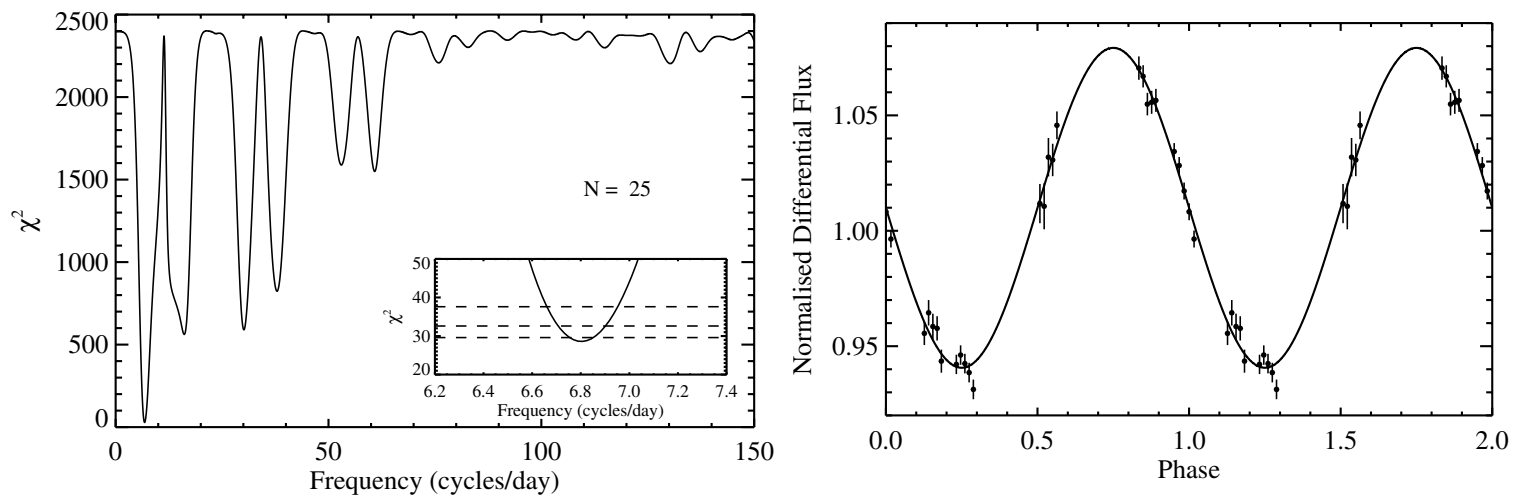

Figure 5. Left: periodogram for PG1031+234. Inset: periodogram zoomed in on the best-fitting frequency of 6.803 cycles day ${ }^{-1}(P=3.53 \mathrm{hr})$. Horizontal dashed lines show the $1 \sigma, 2 \sigma$, and $3 \sigma$ uncertainties. Our measured rotational period is not consistent with the period derived from variability in the optical polarization (Schmidt et al. 1986) of $3.4 \mathrm{hr}$, but we note that caution should be taken, as our period is based on only 25 datapoints. Right: light curve for PG1031+234, folded on our measured period of $3.53 \mathrm{hr}\left(7.06\right.$ cycles day $\left.{ }^{-1}\right)$.

evidence of periodic variability due to the low signal-to-noise ratio ( $\mathrm{S} / \mathrm{N} \sim 26$ ) of our data. In 2003 May the observing conditions were much better, and we more than doubled our exposure times, enabling us to detect variability in this target with a full amplitude of $\sim 2 \%$ and a period of $1.79_{-0.16}^{+0.25} \mathrm{hr}$ (Figure 3 ).

\subsection{3. $P G 1015+015(W D 1015+014)$}

Wickramasinghe \& Cropper (1988) reported a rotational period of 98.7 minutes for the H-rich magnetic white dwarf PG1015+015, based on variations in circular polarization. We find that PG1015+015 is also photometrically variable at a level of $\sim 4.5 \%$ peak-to-peak, with a period of $105_{-7}^{+13}$ minutes (Figure 4). This is consistent with the previous value to within $1 \sigma$. Light curves folded on both 98.7 and 105 minutes look virtually identical, and clearly show the variability in the star (Figure 4). A phase-resolved spectro-polarimetric study of PG1015+015 has been undertaken by Euchner et al. (2006), which suggests that the field varies from 50 to $90 \mathrm{MG}$ across the surface, with strong peaks at 70-80 MG and a photospheric effective temperature $T_{\text {eff }}=10,000 \mathrm{~K} \pm 1000 \mathrm{~K}$.

\subsubsection{PG1031+234 (GH Leo, WD1031+234)}

Schmidt et al. (1986) reported that the optical light from the strongly (500-1000 MG) magnetic DA white dwarf PG1031+234 shows a high degree of circular polarization $\left(P_{\max } \simeq 6 \%, V_{\max } \simeq 12 \%\right)$ and a strong modulation with a period of $3.4 \mathrm{hr}$, which they interpret as the rotational period.
Our results indicate that PG1031+234 is indeed highly variable photometrically, with a full amplitude of $\sim 15 \%$, but we find the period to be $3.53 \pm 0.05 \mathrm{hr}$ (Figure 5). We note that caution should be taken, however, as our period is based on only 25 datapoints.

\subsubsection{PG1533-057 (WD1533-057)}

PG1533-057 was observed during all three epochs of our survey, but most of the data (326 points out of 413) were taken in 2003 May. The periodograms for 2003 May alone, and all of the data combined, show a significant minimum at a frequency of $\approx 12.7$ cycles day ${ }^{-1}$, but the latter shows some aliasing structure. We believe that this is almost certainly due to low-level variability in the comparison stars over the year, which showed a change in average flux of $2 \%$ with respect to each other over the year. This trend was not seen in the shortterm data. For the derivation of the rotational period, and when plotting the folded light curve, we use the data from 2003 May alone. We find that PG1533-057 is a new variable magnetic white dwarf, with $P=1.890 \pm 0.001 \mathrm{hr}$ and a full amplitude of $1.4 \%$ (Figure 6 ).

\subsection{Targets Displaying Variability, but No Determined Period}

For many of our targets we observe variability over the course of an observing run, but did not obtain enough data to unambiguously determine a period. 

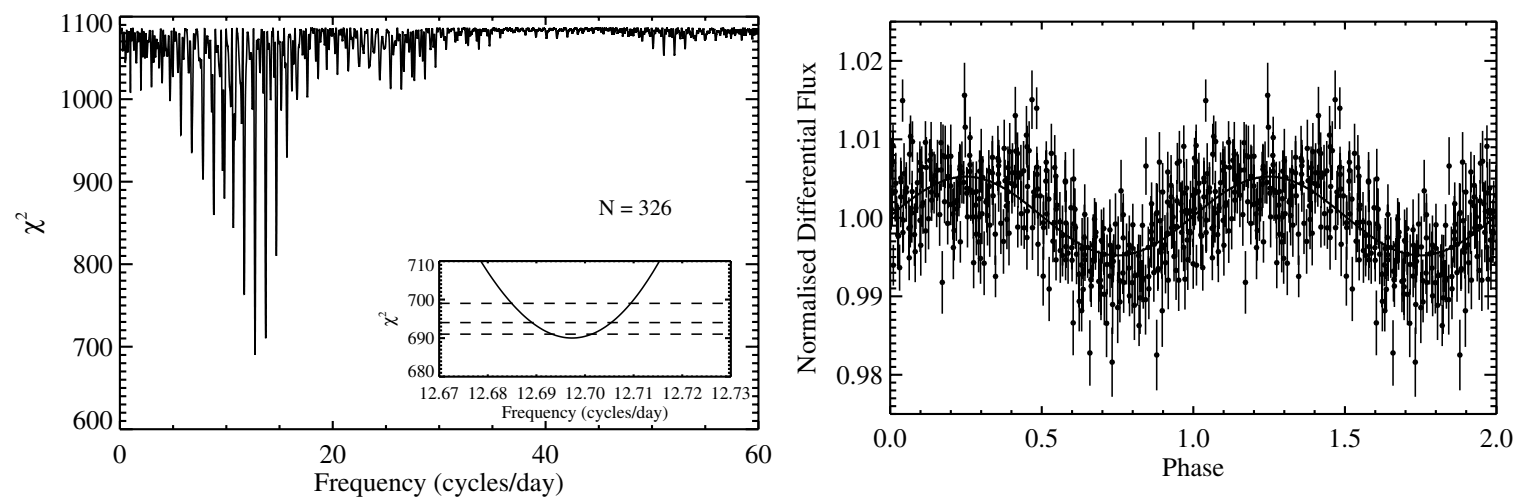

Figure 6. Left: periodogram for PG1533-057, based on the 2003 May data. Inset: the same periodogram, zoomed in on the best-fitting frequency of 12.7 cycles day ${ }^{-1}$ $(P=1.89 \mathrm{hr})$. The horizontal dashed lines indicate the $1 \sigma, 2 \sigma$, and $3 \sigma$ uncertainties. Right: light curve for PG1533-057, based on the 2003 May data, and folded on the best-fitting period of $1.89 \mathrm{hr}$.
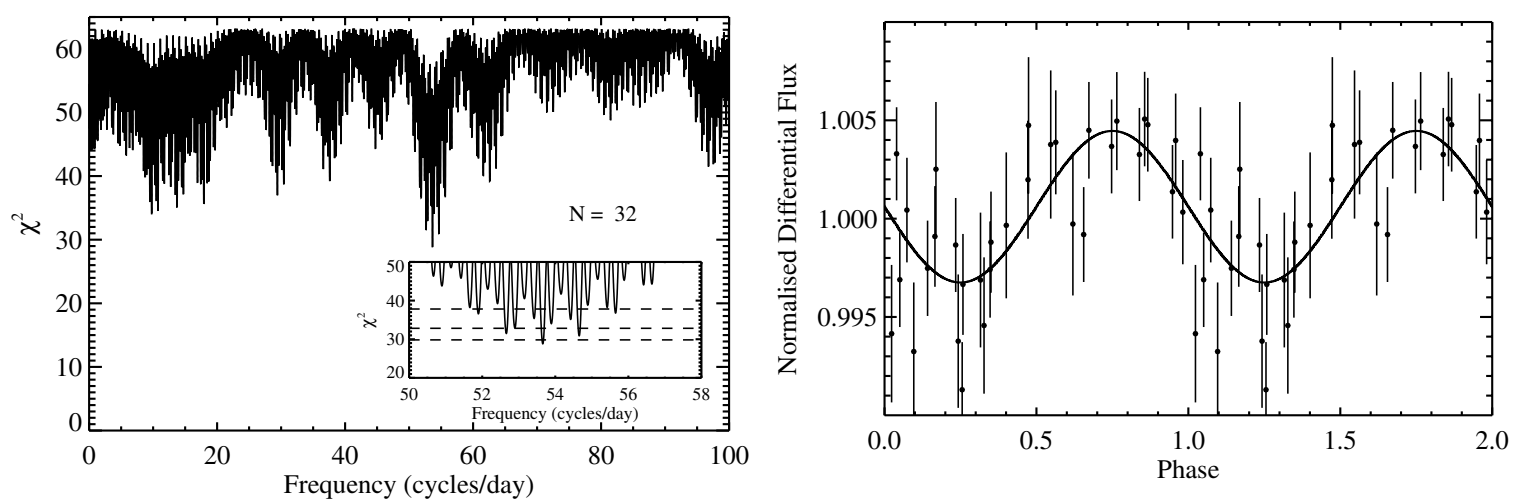

Figure 7. Left: periodogram for G99-47. The best-fitting frequency is at 53.65 cycles day $^{-1}(P=26.8$ minutes), but there are several local minima within the $2 \sigma$ uncertainties. We find no evidence to support the previously determined period listed in Wickramasinghe \& Ferrario $(2000)$ of $P \sim 1 \mathrm{hr}\left(24\right.$ cycles day $\left.{ }^{-1}\right)$. Right: light curve for G99-47, folded on the best-fitting period of 26.8 minutes and binned by a factor of two.

\subsection{1. $G 99-47(W D 0533+053)$}

Bues \& Pragal (1989) reported a possible rotational period for G99-47 of $\sim 1 \mathrm{hr}$, although we have been unable to find any further reference to this in the literature. Our first night of data on this object was affected by strong differential extinction, and so this was discarded in favor of the other three nights of data. The periodogram for G99-47 is shown in Figure 7 and shows several minima within $\Delta \chi^{2}=4(2 \sigma)$ of the best-fitting frequency. We find that the period is probably between 26 and 27.5 minutes, with the minimum in the periodogram occurring at a frequency of 53.65 cycles day ${ }^{-1}(P=26.8$ minutes $)$. The folded light curve on this best-fitting period (same figure) shows that the amplitude of the variability is very low-approximately $1 \%$ peak-to-peak.

\subsubsection{G217-037 (WD0009+501)}

Schmidt \& Smith (1995) discovered the magnetic nature of G217-037 and reported spectropolarimetric variability on a timescale of 2-20 hr. We observed G217-037 over a total of five nights in 2002 August. The light curve is clearly variable, but we have been unable to find the period. The global minimum in the periodogram suggests a frequency of 0.47 cycles day $^{-1}$ $(P=2.13$ days), but folding the light curve on this period shows that it is dominated by the large jump in flux between the first and third nights. In an attempt to check for other periods in the data, we masked the first night and the noisy datapoints from the last night, and re-ran the periodogram on the remaining four nights of data. The new minimum falls at 2.90 cycles day $^{-1}(P=8.26 \mathrm{hr}$;

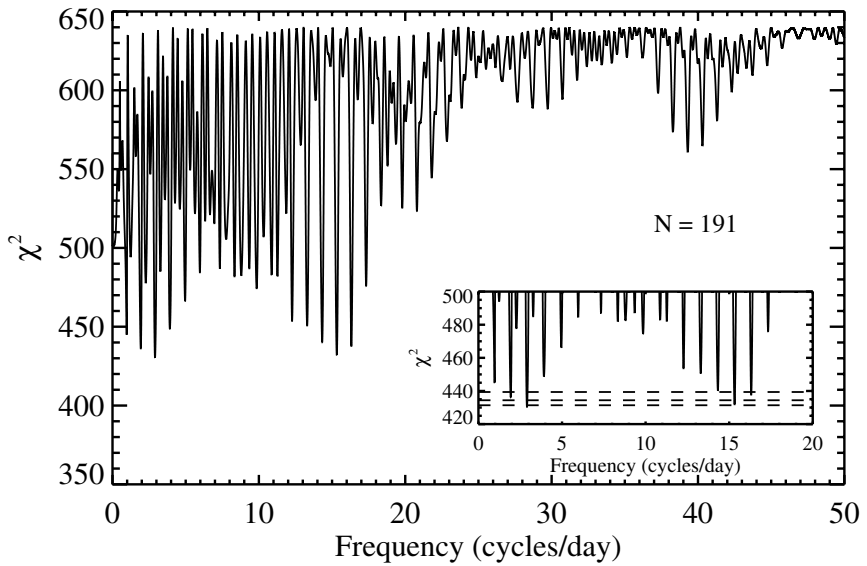

Figure 8. Periodogram for G217-037, for the last four nights of data. The best-fitting period for the full periodogram (not shown) was at $P=2.13$ days, but the folded light curve (Figure 9) showed that this was dominated by a jump in flux between night 1 and night 3 . The periodogram for the last four nights of data (plotted here) gives a global minimum at $P=8.26 \mathrm{hr}$, which is consistent with the spectropolarimetric period of $\sim 8 \mathrm{hr}$ reported by Valyavin et al. (2005).

Figure 8), which is consistent with the spectropolarimetric period of $\sim 8 \mathrm{hr}$ reported by Valyavin et al. (2005), but there are a number of minima within $\Delta \chi^{2}=4(2 \sigma)$ of the global minimum. There was no change in the FAP between the full dataset and the masked dataset. The folded light curve is shown in Figure 9. We conclude that this target is variable over a week, but we have been unable to unambiguously determine the period. 

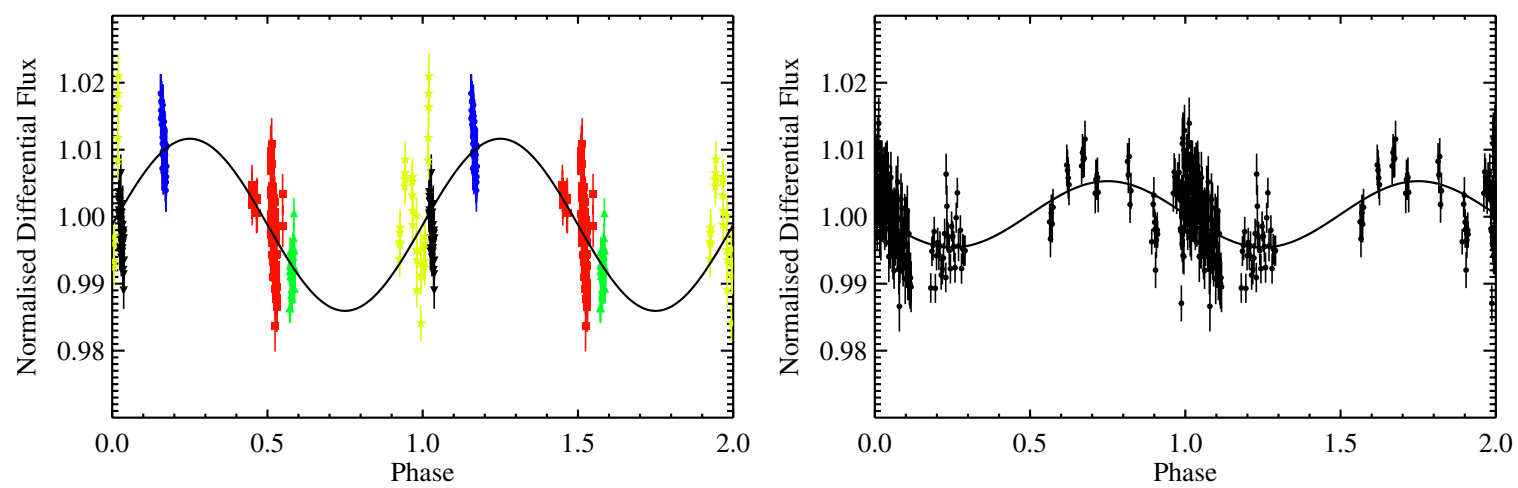

Figure 9. Left: the data for G217-037, taken over five nights, folded on the best-fitting period for all of the data of $P=2.13$ days. The nights have been color-coded, with night 1 blue, night 2 green, night 3 black, night 4 red, and night 5 yellow. The period is dominated by the jump in flux between nights 1 and 3 . Right: the light curve folded on the best period from just the last four nights $(P=8.26 \mathrm{hr}$ ). This is consistent with the spectropolarimetric period reported by Valyavin et al. (2005).

(A color version of this figure is available in the online journal.)
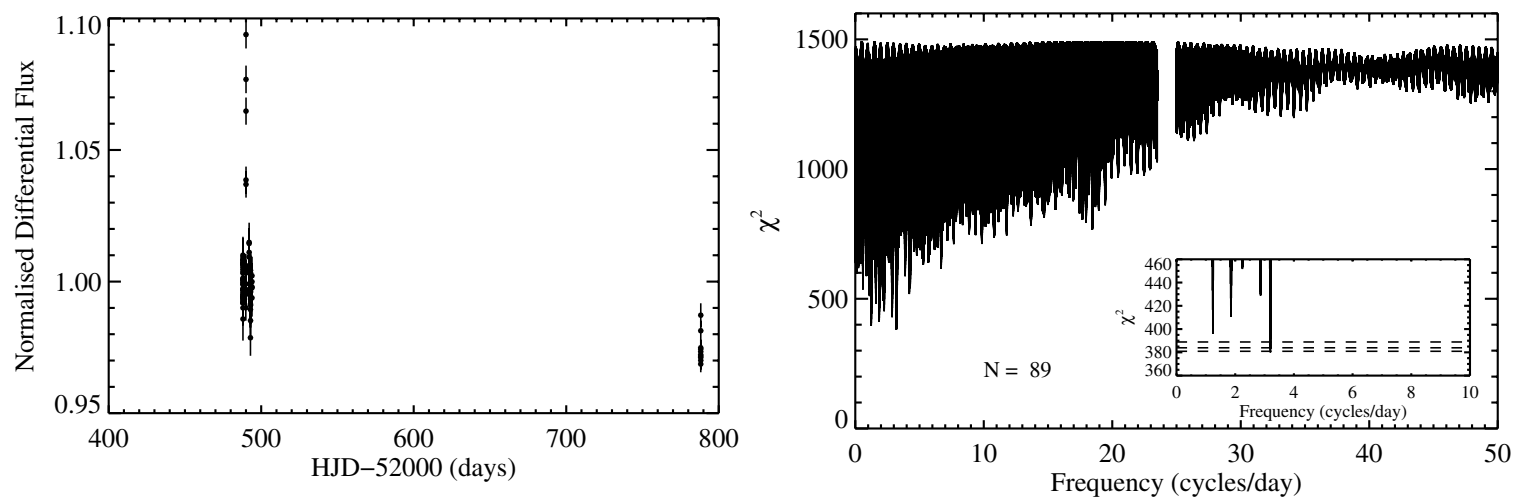

Figure 10. Left: the data for G227-28, showing a drop of 3\% in the differential flux over the year. Right: the periodogram for G227-28 shows a significant global minimum at 3.19 cycles day $^{-1}(7.5 \mathrm{hr})$, but the folded light curves (Figure 11) do not support a short-term periodicity.
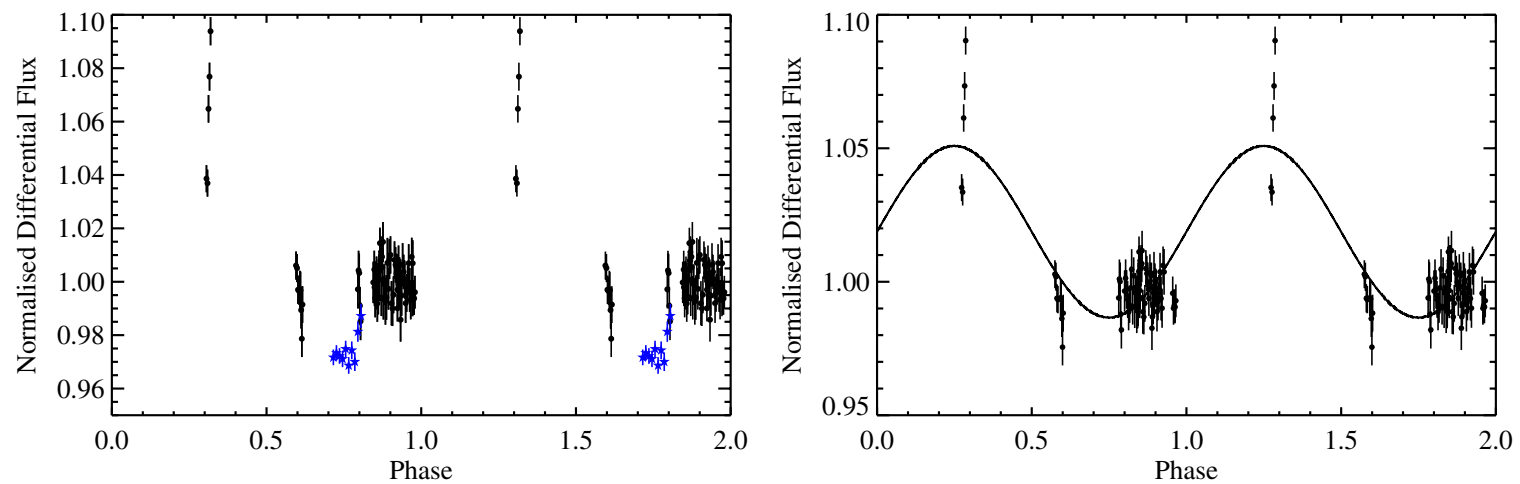

Figure 11. Left: all of the data on G227-28, folded on the best period of $P=7.5 \mathrm{hr}$. Data from the first epoch are in black, and from the second are shown in blue. Right: August data alone, folded on the best period for that dataset, of $P=1.31$ days.

(A color version of this figure is available in the online journal.)

\subsubsection{G227-28 (WD1820+609)}

We find no evidence for variability in this object over short timescales, but find that there is a drop of $3 \%$ in the differential photometry over the year (Figure 10). We have been unable to determine a period (Figure 11), so simply conclude that this target is probably varying over timescales of $\sim$ months-years with full amplitude of $3 \%$ or more. We are conducting follow-up observations to confirm these long-term modulations.

$$
\text { 3.2.4. G240-72 (WD1748+708) }
$$

Berdyugin \& Pirola (1999) reported extremely slow variability in the polarization of this object over several decades, which they interpret as a rotational period of $\geqslant 100 \mathrm{yr}$. We find no evidence for short-term variability in this star: the rms of the scatter in the light curve is lower than the rms of the scatter in the comparisons. We do, however, see an increase in the differential flux of $\sim 2.5 \%$ over the 10 months between 2002 August and 2003 May (Figure 12), well above the rms level of the comparisons, and the $\chi^{2}$ of the best-fitting period is $\Delta \chi^{2}=1382$ better than for a constant fit. The periodogram is dominated by the scatter at short periods, so we have not included it here. We also ran a periodogram on the August data alone to check for short-term periodicity buried in the scatter. We found no significant minima below the Nyquist frequency, and the $\chi^{2}$ of a constant fit 


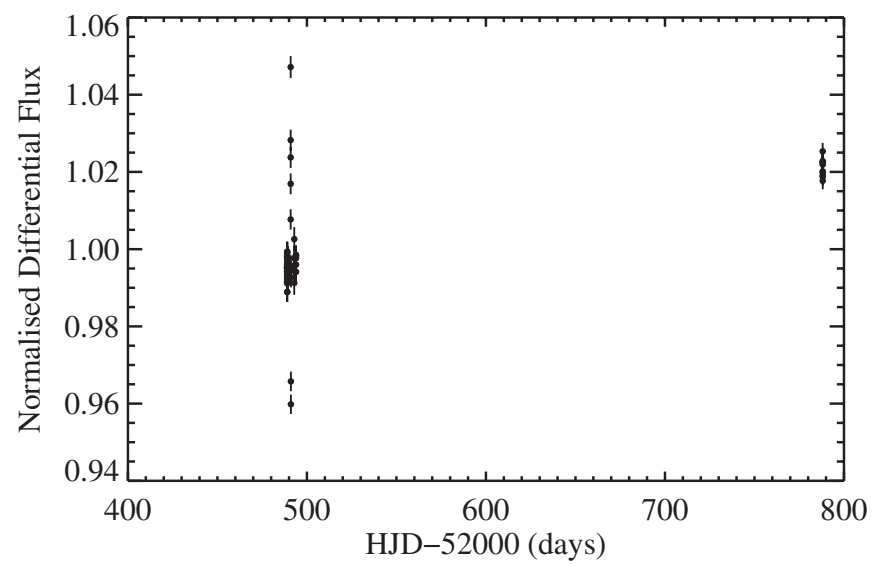

Figure 12. Data for G240-72, taken in 2002 August and 2003 May. The scatter in the comparison star photometry is greater than the scatter in the target photometry over periods of a week, but the $\sim 2.5 \%$ variability over a few months is significantly greater than the rms of the comparison photometry. We find no evidence for variability over a week, but conclude that there is strong variability over a few months.

is 51 over 52 dof. We conclude that there is no evidence for short-term variability, but that this object is seen to vary over 10 months. These conclusions are supported by the FAPs, with $\mathrm{FAP}<0.001$ for the long-period variability, but $\mathrm{FAP}=0.443$ over the short term.

\subsection{5. $L B 8915(P G 0853+164)$}

This white dwarf was wrongly identified in Putney (1997) and Wesemael et al. (2001) as LB8827. Putney (1997) discovered LB8915 to be magnetic with an effective mean line-of-sight field component $B_{e}=-1000 \pm 500 \mathrm{kG}$. Wesemael et al. (2001) classified LB8915 as a mixed H/He white dwarf, but noted that on three occasions they measured a weaker field strength and opposite sign of that reported in Putney (1997; $B_{e}<100 \mathrm{kG}$ ), which suggests variability and rotation. They also noted the Balmer line strength varies with time, suggesting $\mathrm{H} / \mathrm{He}$ atmospheric irregularities over the surface. At $T=$ 21,000-28,000 K, LB8915 may lie within the DB instability strip. A search for pulsations over a baseline of $3 \mathrm{hr}$ proved negative, although Wesemael et al. (2001) note they cannot rule out variability at periods of $2 \mathrm{hr}$ or greater. We have discovered variability in LB8915 at a peak-to-peak level of about $4 \%$. The periodogram displays a heavy aliasing structure, which has made it difficult to select a best-fitting period. For the floating-mean periodogram, the global minimum occurs at a frequency of 2.11 cycles day ${ }^{-1}(P=11.3 \mathrm{hr})$, with a $\chi^{2}$ of 25 over 27 dof compared to a constant fit with $\chi^{2}=426$ over 29 dof (Figure 13). The light curve folded on this period looks similar to the next three best-fitting frequencies, at 9.18, 1.04 , and 4.17 cycles day $^{-1}$. We therefore also tested the data using a Lomb-Scargle periodogram, which showed a change in the best period, giving the two best-fitting frequencies as 9.18 $\left(\chi^{2}=25\right.$ over 27 dof $)$ and 4.17 cycles day $^{-1}\left(\chi^{2}=28 / 27\right.$ dof $)$. We conclude that LB8915 is variable, with a period between 2 and $24 \mathrm{hr}$. Light curves folded on the two best-fitting frequencies of 2.11 and 9.18 cycles day ${ }^{-1}(P=11.3 \mathrm{hr}$ and $2.6 \mathrm{hr}$ respectively) are shown in Figure 14.

\subsubsection{LHS2273 (WD1026+117)}

We observed LHS2273 over five nights during 2003 May. We find that LHS 2273 is variable well above the level in

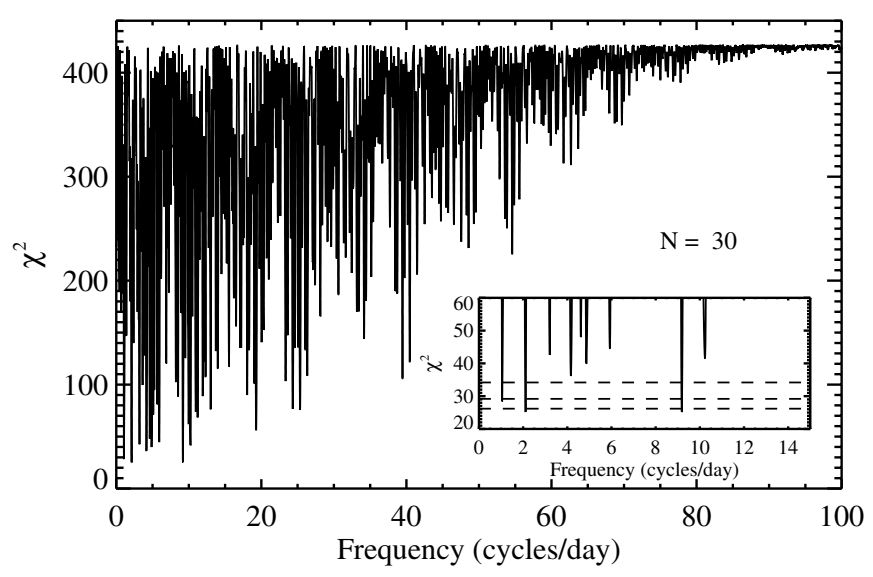

Figure 13. Floating fit periodogram for LB8915. The global minimum is at 2.11 cycles day ${ }^{-1}(P=11.3 \mathrm{hr})$ with the second best frequency at 9.18 cycles day ${ }^{-1}(2.6 \mathrm{hr})$.

the comparison stars, and the $\chi^{2}$ in the periodogram is an improvement of $\Delta \chi^{2}=19$ over a constant fit. The FAP is 0.016 , which is slightly above our significance cutoff, but the amplitude of the folded light curve is incredibly low, making it likely that the FAP will flag this as a false alarm, and the other evidence points strongly toward variability in this object. The periodogram shows several peaks within the $3 \sigma$ uncertainties on the global minimum. Folding on the best period of 40.86 minutes (35.24 cycles day ${ }^{-1}$ ) gives the folded light curve shown at the bottom of Figure 15. We conclude that LHS2273 is probably varying, with a period around 35-45 minutes.

\subsubsection{LHS5064 (WD0257+080)}

We observed LHS5064 over five nights in 2003 February and have discovered significant photometric variability in the star, on a level of about $4.5 \%$ peak-to-peak. The best-fit period is 1.151 cycles day $^{-1}\left(\chi^{2}=36\right.$ over 30 dof $)$, corresponding to a period of $20.85 \mathrm{hr}$ (Figure 16). This is an improvement of $\Delta \chi^{2}$ of 340 compared to a constant fit. A Lomb-Scargle periodogram gives the same best-fitting period. When folded on this period, the light curve looks approximately sinusoidal, although there are a number of other periods around the best-fit value that are within the $3 \sigma$ uncertainties. We conclude that LHS5064 is variable, with a period of between $9 \mathrm{hr}$ and 6 days, but a most likely period of $\sim 20 \mathrm{hr}$.

\subsection{8. $P G 1658+441$}

PG1658+441 was observed during 2002 August and again, briefly, in 2003 May. We find that the star is variable above the level in the comparison stars, and the $\chi^{2}$ of a constant fit is 363 over 63 dof, while the global minimum in the periodogram has a $\chi^{2}$ of 77 over 60 dof (Figure 17). We find that there are several peaks in the periodogram within $3 \sigma$ of the $\chi^{2}$ minimum. When folding the light curve on these periods, however, we find that they all rely on a shift in the mean flux between the two datasets, seen in the clumping of the data from the two epochs, and therefore probably do not represent a real rotational period. We therefore simply conclude that PG1658+441 is probably variable with a period between $6 \mathrm{hr}$ and 4 days.

$$
\text { 3.2.9. } P G 2329+267
$$

PG2329+267 was observed over the week in 2002 August, and again, briefly, in 2003 May to check for long-term variability. The global minimum in the periodogram is the same 

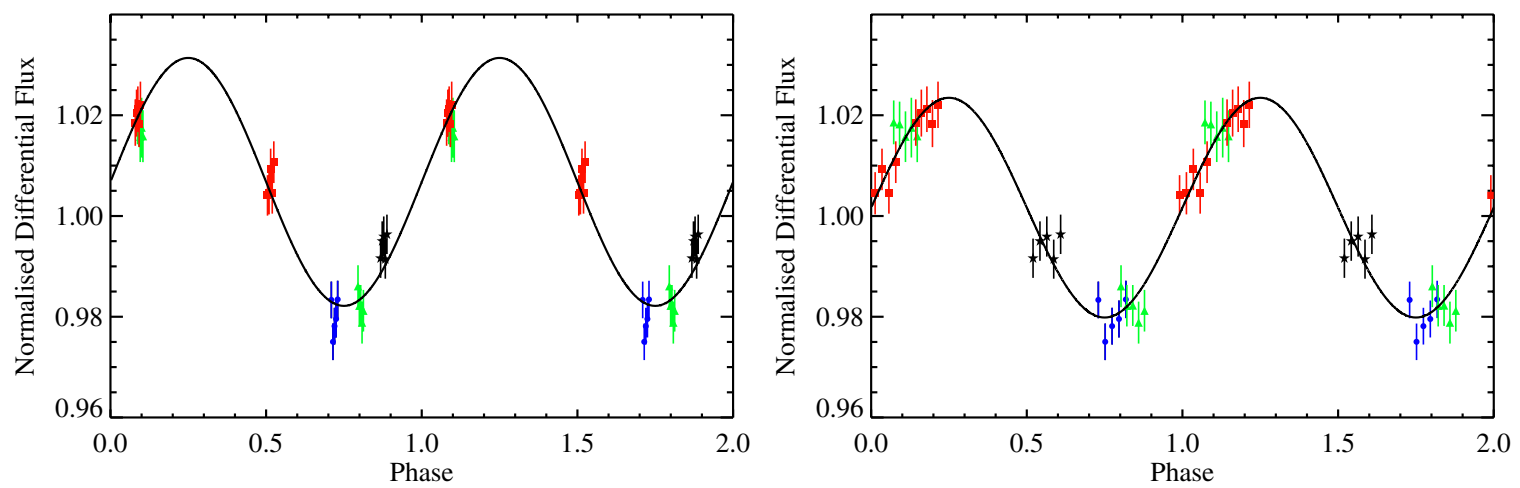

Figure 14. Phase-folded light curves for LB8915. Left: period $=11.3 \mathrm{hr}$. Right: $P=2.6 \mathrm{hr}$. Different nights are plotted in different colors. (A color version of this figure is available in the online journal.)
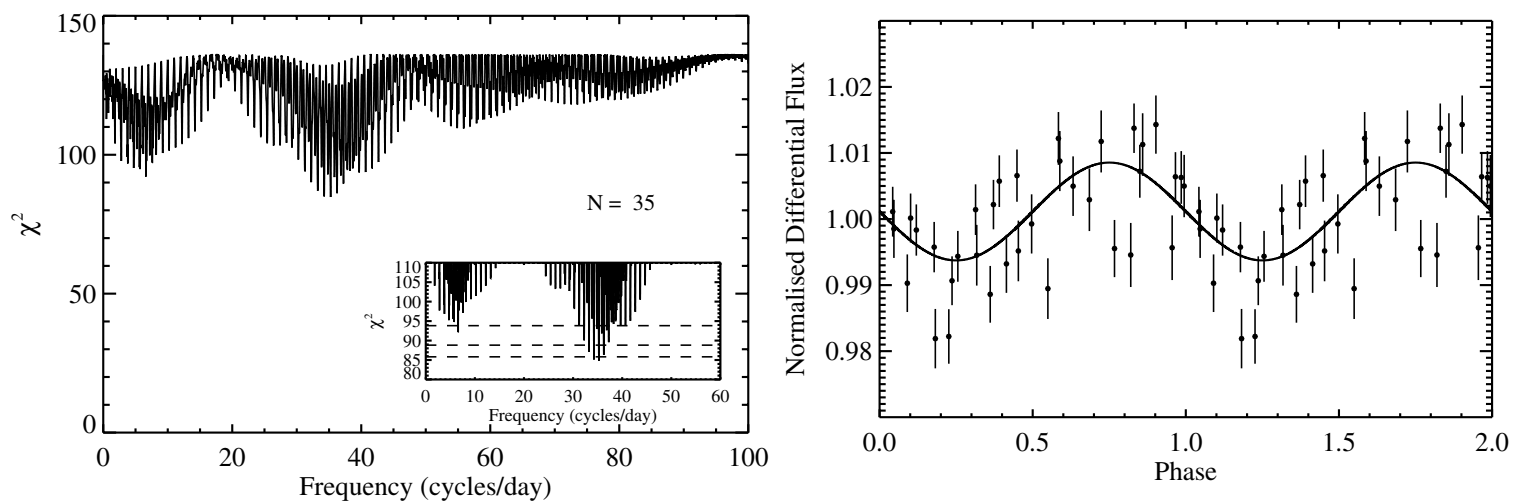

Figure 15. Left: periodogram for LHS2273. Right: light curve folded on the best period of 40.9 minutes.
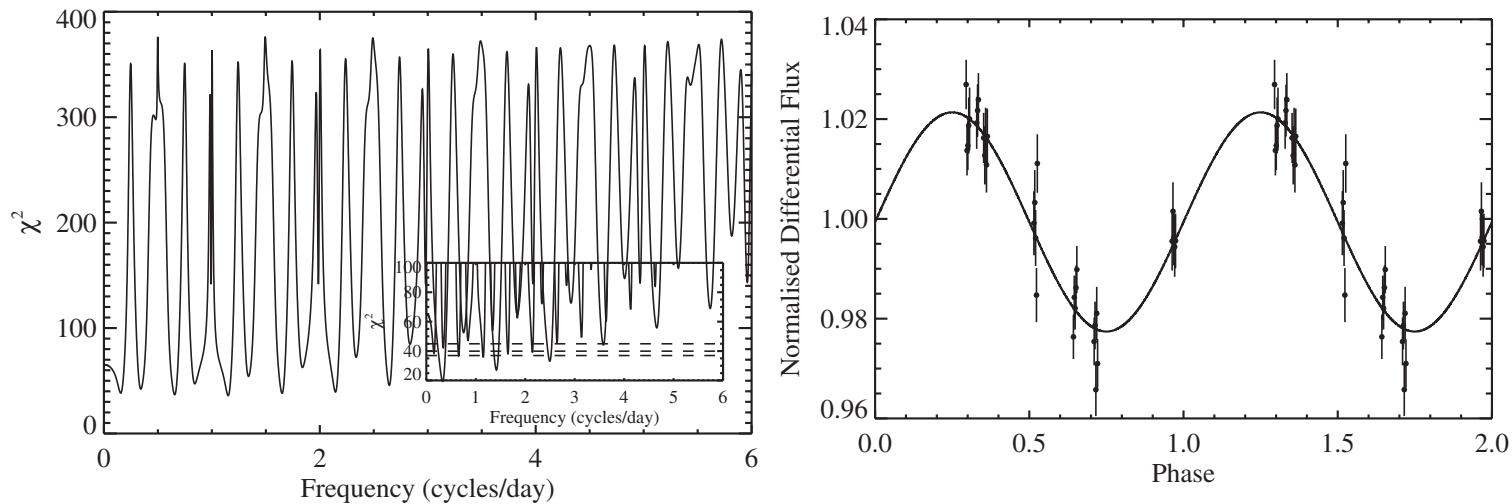

Figure 16. Left: periodogram for LHS5064. While the best frequency is at 1.151 cycles day ${ }^{-1}$, there are a number of other signals within the $3 \sigma$ uncertainties. Right: light curve folded on the best period of $20.85 \mathrm{hr}$.

for all the data, and the August data alone, at a frequency of 8.6745 cycles day ${ }^{-1}(2.767 \mathrm{hr})$. A fit at this frequency gives a $\chi^{2}=458$ over 150 dof compared to $\chi^{2}=707$ over 152 dof for a constant fit. The light curve folded on the best period of $2.767 \mathrm{hr}$ is shown in Figure 18; however, we are cautious in claiming that this is the definitive period for this star, since there are two other minima within $3 \sigma$ of the best fit.

\subsection{Targets Displaying No Clear Evidence of Variability}

\subsubsection{Targets with Variable Comparisons}

A number of the white dwarfs were found to have periodic variability in the comparison stars on level equal to or greater than the modulation detected in the target. We therefore conclude that we have no evidence for variability in these targets and exclude them from further analysis and discussion. White dwarfs falling into this category are: EUVE J1439+75.0, G99-37, G141-2, G227-35, G234-4, G256-7, GD77, GD229, LP907-037, and SBSS1349+545. A further target, G195-19, had only one comparison star (the other was saturated), and so we have no way of knowing whether the variability detected in this target on a level of $\sim 4 \%$ was due to the target or the comparison.

\subsubsection{Targets Showing No Significant Variability}

Many of our targets were either too faint or too sparsely observed to reliably measure their variability. Others showed no variability above the scatter in the comparison stars. One showed evidence of variability, but the FAP was very high. They are described below, but are not included in a subsequent 

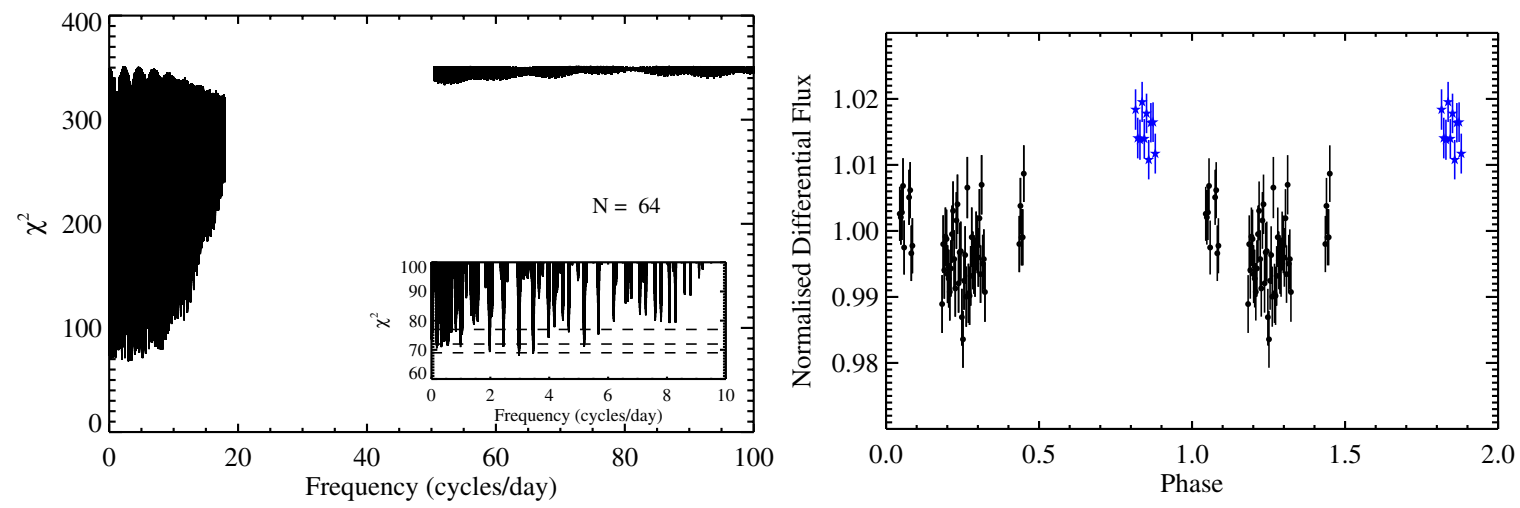

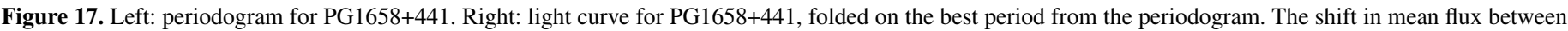

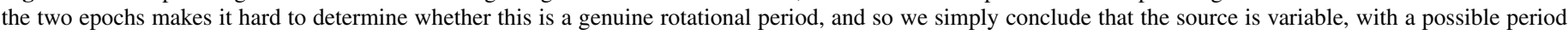
between $6 \mathrm{hr}$ and 4 days.

(A color version of this figure is available in the online journal.)
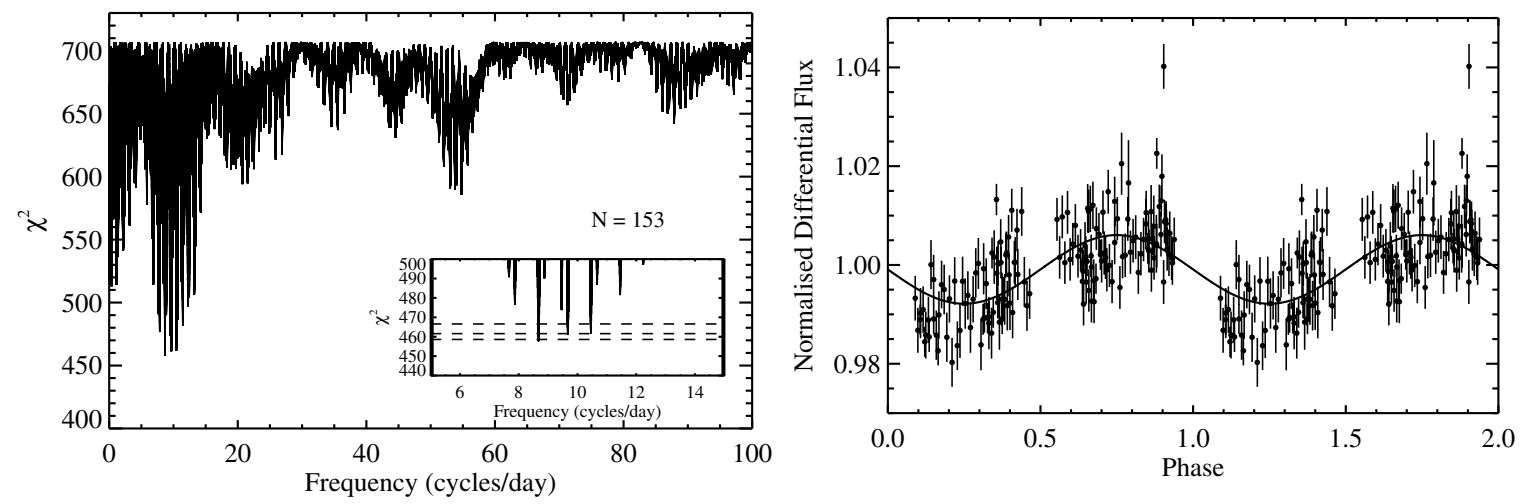

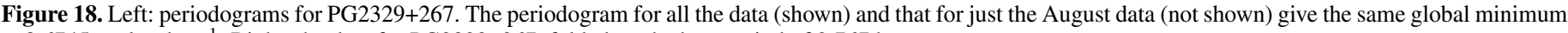
at 8.6745 cycles day $^{-1}$. Right: the data for PG2329+267, folded on the best period of $2.767 \mathrm{hr}$.

analysis. Some have been included in our long-term follow-up study to search for variability on timescales of months-years.

$$
\text { 3.3.2.1. G158-45 (WD0011-134) }
$$

Putney (1997) reported polarimetric variations in G158-45, suggesting a rotational period of between $11 \mathrm{hr}$ and a few days. We observed the system over six nights during the August run, and found tentative evidence for photometric variability in the star with a period between 30 minutes and a few days and a peak-to-peak amplitude of $\sim 1 \%$. The global minimum at $P=$ $1.44 \mathrm{hr}$ has a $\chi^{2}$ of 112 over 79 dof, which is significantly better than a constant fit with $\chi^{2}$ of 142 over 81 dof. It should be noted that G158-45 is at the faint end of our sample (15.9 mag), and so the uncertainties on the data points are relatively large ( $\pm \sim 0.75 \%$; Figure 19). Despite the evidence for variability in this object, the FAP is very high, at 0.785 , so we are unable to include G158-45 in the list of variable sources. This target is included in our follow-up study sample.

$$
\text { 3.3.2.2. } G 183-35 \text { (WD1814+248) }
$$

Putney (1997) reported possible rotation of this object with a period between 50 minutes and a few years. From our photometric data, taken over a week in 2002 August and a further week in 2003 May, we find no evidence of rotation on timescales of less than a year. A constant fit to the data gives a $\chi^{2}$ of 122 over 152 dof, compared to a $\chi^{2}$ minimum in the periodogram of 101 over 149 dof (Figure 20), but, as these numbers suggest, the uncertainties on the data points are too large for this to be meaningful. A light curve folded on the periodogram global minimum shows that the data are flat over the year. We conclude that G183-35 is not varying at the 4\% peak-to-peak level on timescales of less than a year, and are following up this object on longer timescales.

\subsubsection{GD90 (WD0816+376)}

Due to poor observing conditions, we only took 16 observations of GD90 during the 2003 February run. While the difference between a constant and sinusoidal fit formally suggests variability $\left(\Delta \chi^{2}=23\right)$, there was no significant global minimum in the periodogram (the minimum was at a period of 7.7 minutes; Figure 21). We rule out variability at the $4 \%$ peakto-peak level, and conclude that more data are required on this object.

\subsubsection{Grw $+70^{\circ} 8247(W D 1900+70)$}

The polarization curves for this star have remained unchanged over $25 \mathrm{yr}$ of observations, suggesting that this star is a very slow rotator, with a period of $\geqslant 100 \mathrm{yr}$. We took data over the week of the 2002 August run, plus one set during 2003 May to check for any long-term variations over the year. A constant fit to the data has a $\chi^{2}$ of 472 over 148 dof, while the global minimum in the periodogram at 0.248 cycles day ${ }^{-1}$ has $\chi^{2}=318$ over 146 dof (Figure 22). We note that the rms scatter in the comparisons is greater than the scatter in the target flux and conclude that we 

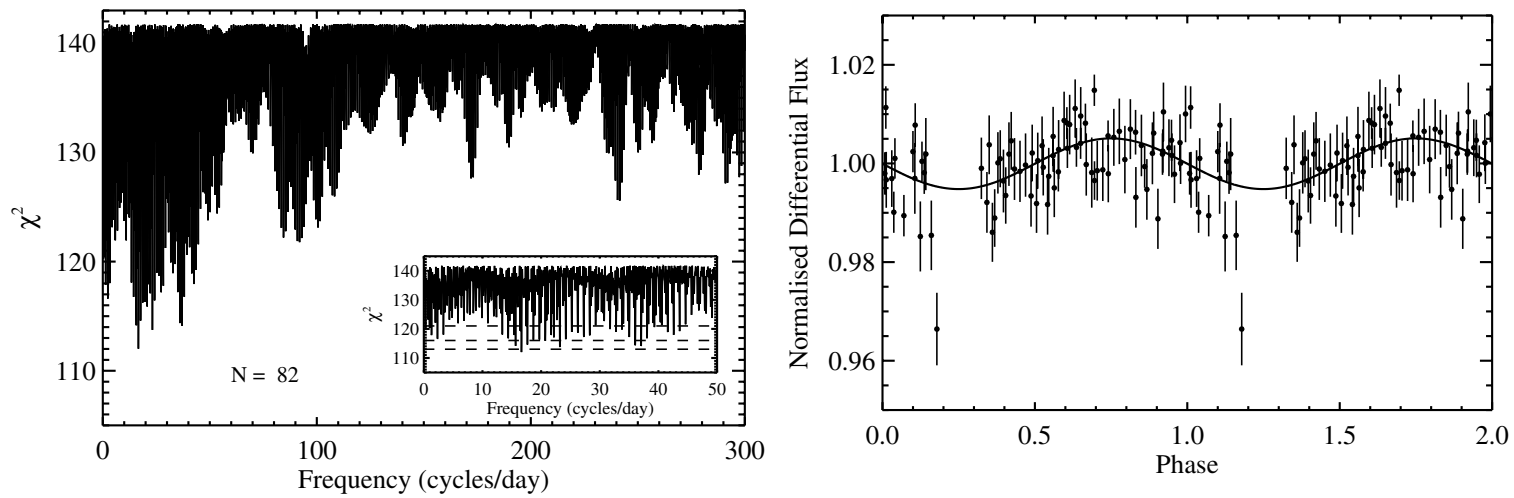

Figure 19. Left: periodogram for G158-45, with the global minimum at a frequency of 16.65 cycles day ${ }^{-1}$. There are a number of minima within $2 \sigma$ of this frequency. Right: light curve for G158-45, folded on the best-fitting period of $1.44 \mathrm{hr}$. The folded light curve looks promising, but the FAP is 0.785 , so we cannot formally classify this as variable. We do, however, note that the amplitude is very low, making it more likely that this will be flagged as a false alarm.
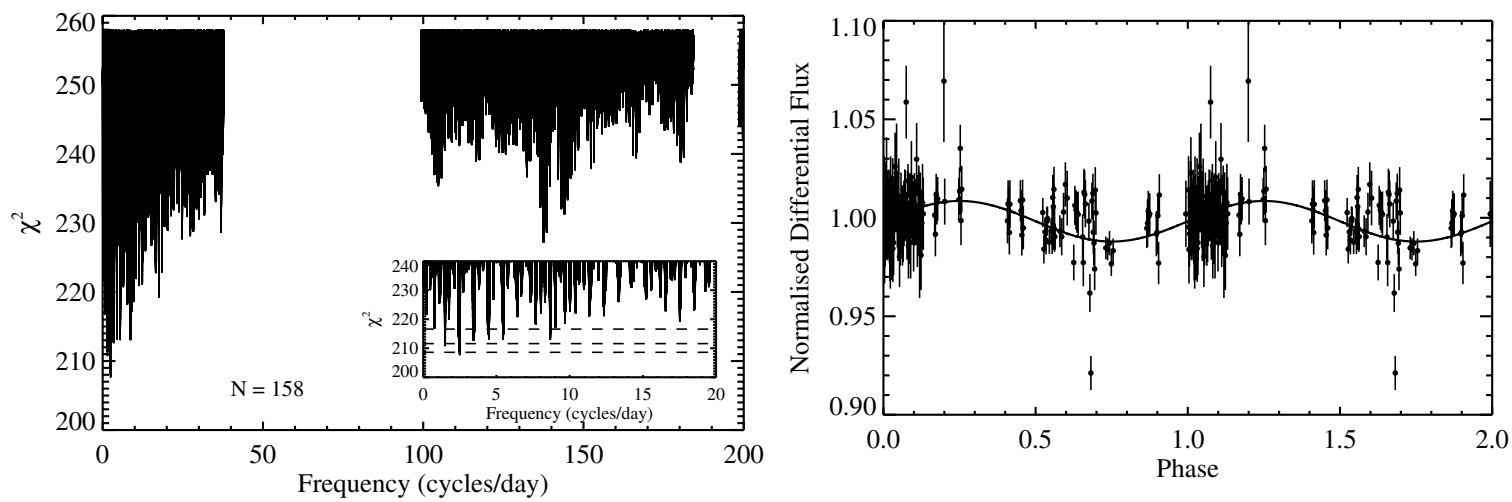

Figure 20. Periodogram and folded light curve for G183-35. The light curve is folded on a period of $8.99 \mathrm{hr}$, but shows significant scatter and no convincing periodicity on the timescales of our observations.

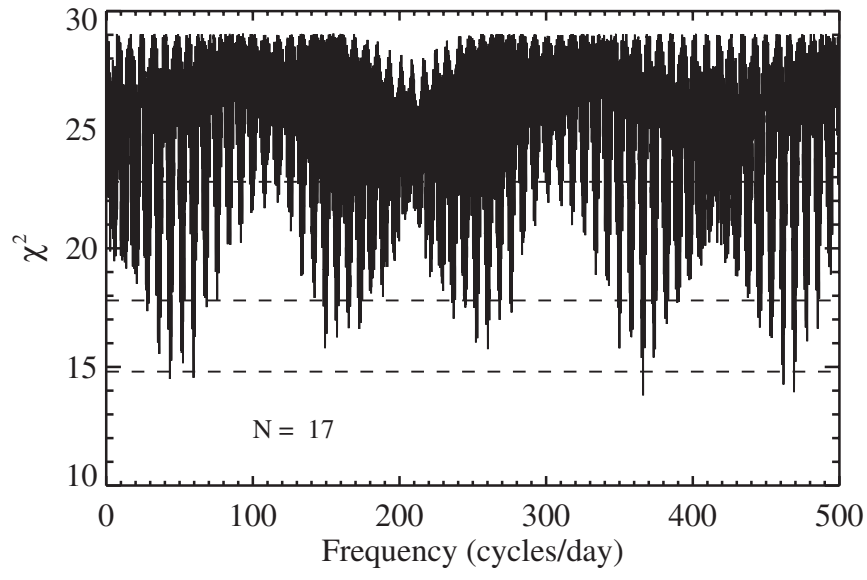

Figure 21. Periodogram for GD90, with no significant minima.

have not found any evidence for periodic variability at the $4 \%$ peak-to-peak level in the target. This target is included in our follow-up program to test for significant long-term variability.

\subsubsection{HE0107-0158}

Reimers et al. (1998) claimed HE0107-0158 was a magnetic white dwarf based on a single low-resolution spectrum, but Schmidt et al. (2001) believe that this system is a non-magnetic white dwarf-cool dwarf pair, and on the basis of this we exclude it from the correlation analysis of this survey. There is no evidence for variability in this system above the $4 \%$ peak-to- peak level. The difference between a sinusoidal fit and a constant fit is only $\Delta \chi^{2}=14.1$, so this fails our variability test (Figure 23).

\subsubsection{HE1045-0908}

Schmidt et al. (2001) report variability in their spectroscopy of HE1045-0908, and suggest a rotational period for the star of between 2 and $4 \mathrm{hr}$. Euchner et al. (2005) place an upper limit on the period of $P<9 \mathrm{hr}$. The target is among the faintest in our survey (only $16.8 \mathrm{mag}$ ), and our data have poor $\mathrm{S} / \mathrm{N}$ and considerable scatter, but we find no evidence for variability greater than the $2 \%$ full-amplitude level on the timescale from the literature. The difference between a constant fit and a sinusoid fails our test for variability $\left(\Delta \chi^{2}=14\right.$; Figure 24).

$$
\text { 3.3.2.7. } L B 11146(W D 0945+246)
$$

LB11146 is a double-degenerate binary system, confirmed by Hubble Space Telescope observations (Nelan 2007), and is therefore excluded from our correlation analysis. We only have 16 data points for this target, find no significant minima when running a periodogram, and the rms scatter of the comparisons is greater than the scatter in the target data. Due to the sparse data, we are unable to rule out variability in this object at any level. (Figure 25).

\subsubsection{8. $P G 0136+251$}

Kawka et al. (2007) list PG0136+251 as a non-magnetic star. We find no evidence for variability in this object above the $4 \%$ 

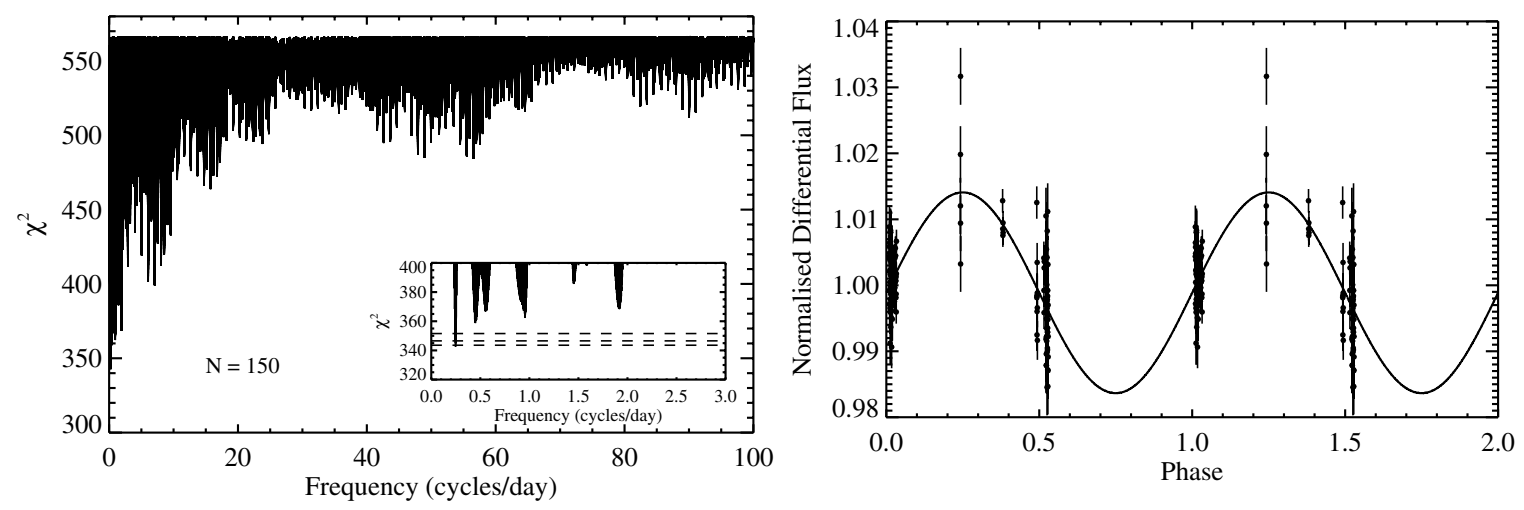

Figure 22. Periodogram and folded light curve for Grw $+70^{\circ} 8247$. The light curve is folded on a period of 4.03 days, but shows significant scatter and no convincing periodicity on the timescales of our observations.

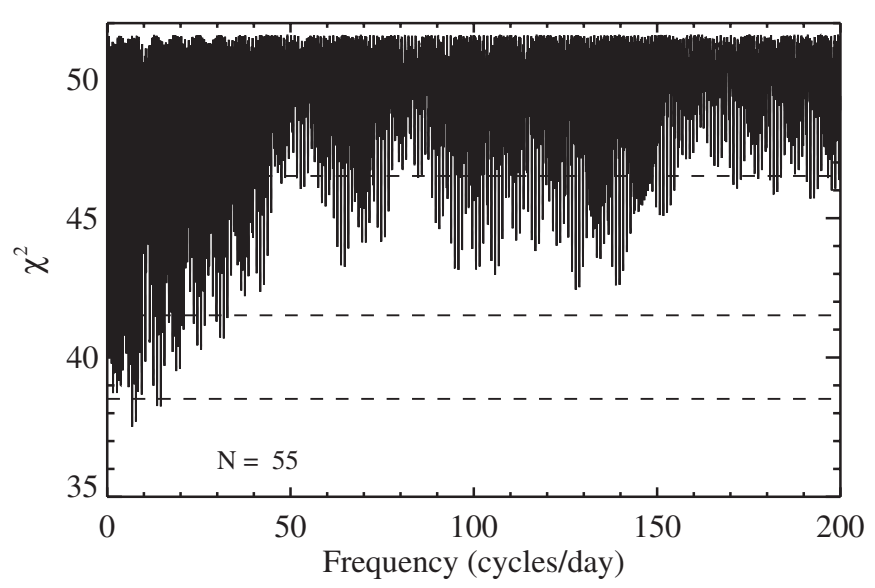

Figure 23. Periodogram for HE0107-0158, with no significant minima.

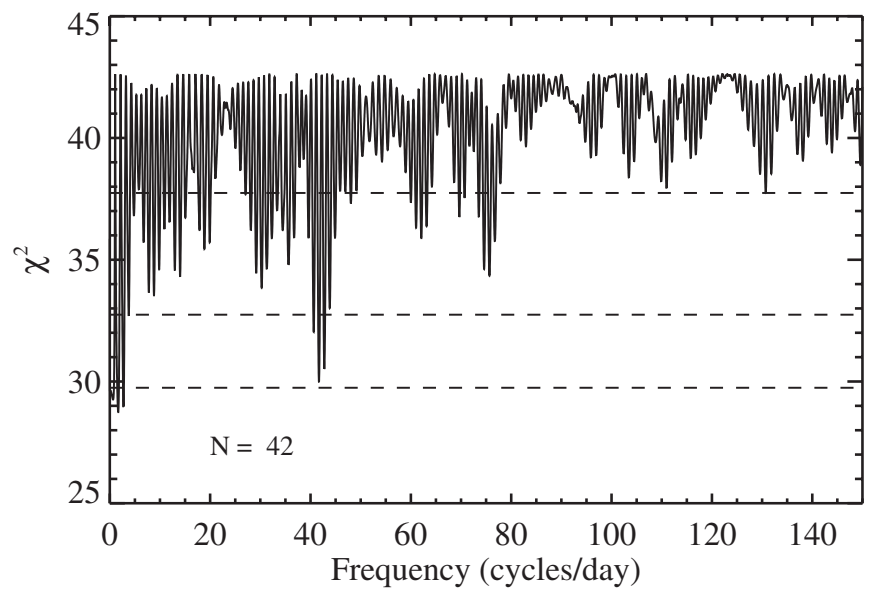

Figure 24. Periodogram for HE1045-0908, with no significant minima.

level. The global minimum in the periodogram is insignificant when compared to a constant fit, with $\Delta \chi^{2}=12$ (Figure 26).

\section{DISCUSSION}

Of our 33 targets, 2 are believed to be binary systems (HE0107-0158, EUVE J1439+75.0), and 1 (PG0136+251) is believed to be non-magnetic (and indeed found here to be nonvariable), and they are therefore excluded from further analysis. Of the remaining 30 isolated magnetic white dwarfs, 5 are

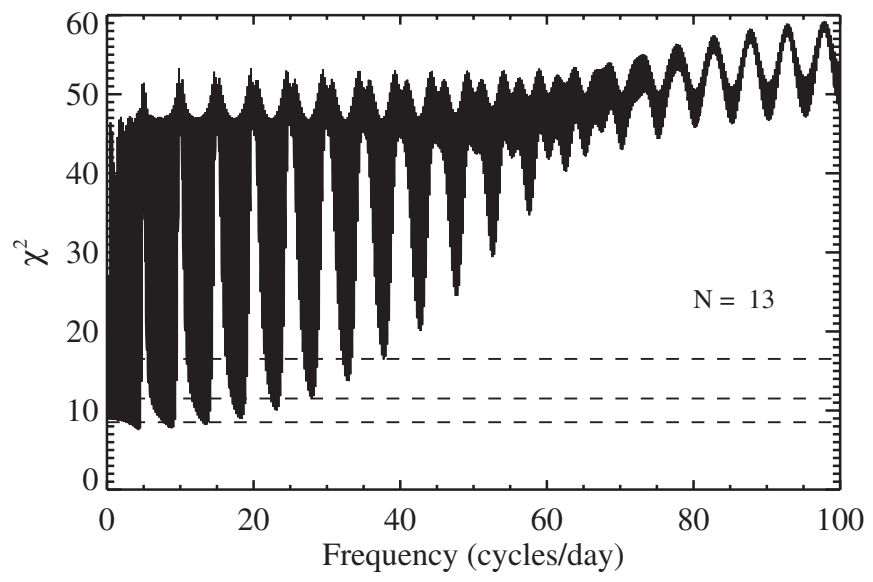

Figure 25. Periodogram for LB11146, with no significant minima.

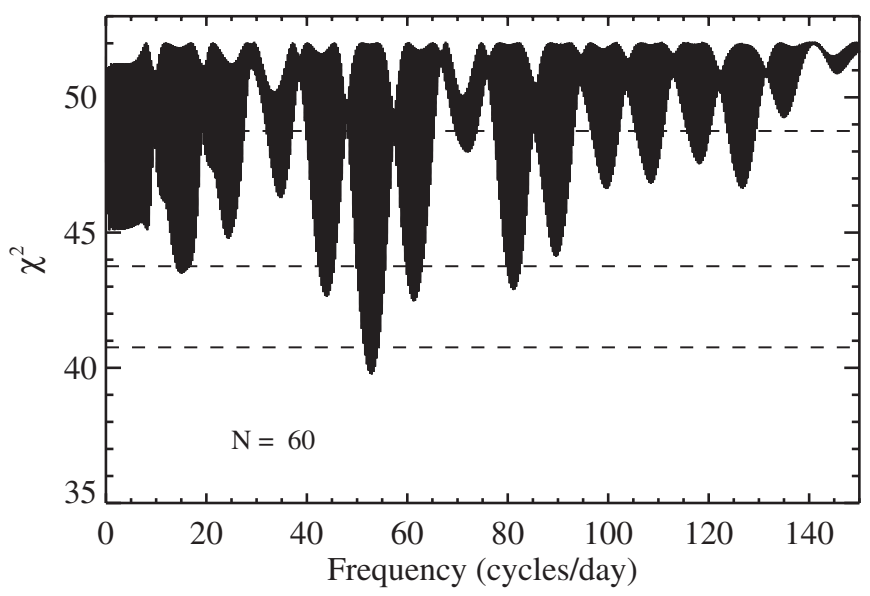

Figure 26. Periodogram for PG0136+251, with no significant minima.

variable with reliably derived rotation periods, and a further 9 are seen to vary during our study, but we were unable to determine the period.

There is no evidence for variability in the remaining 16 objects: 9 had variable comparison stars, 5 require more data, and 2 were not seen to vary above the scatter in the comparisons. The period distribution for the variable magnetic white dwarfs in our sample can be seen in Figure 1. 


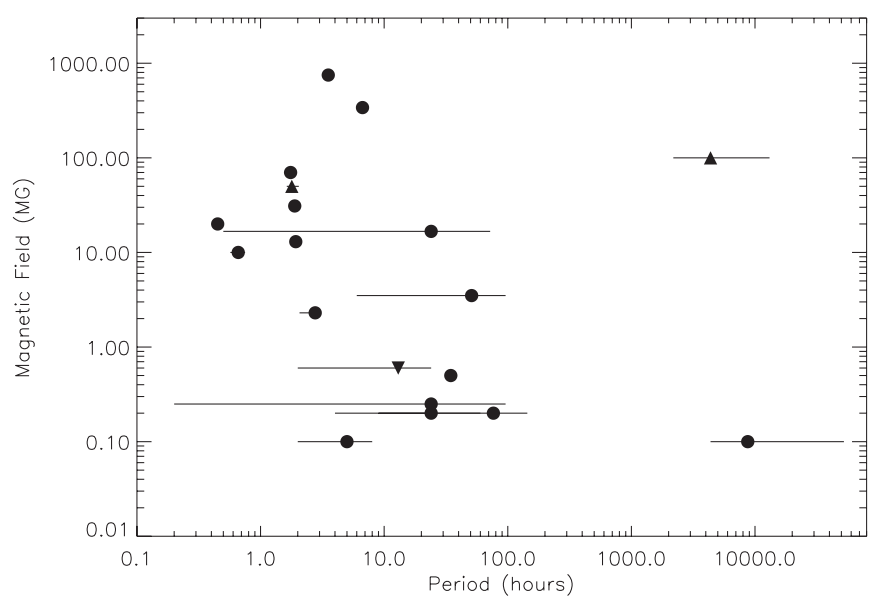

Figure 27. Plot of field strength vs. measured period for our variable white dwarf sample. Also included are WD1953-011 and GD356, from Brinkworth et al. $(2004,2005)$. The shape of the plotting symbols describes the white dwarf composition-DA white dwarfs are plotted as circles, DB white dwarfs as upright triangles, while targets that have been reported as both DA and DB are plotted as upside-down triangles. We find no correlation between field strength and spin period in our sample.

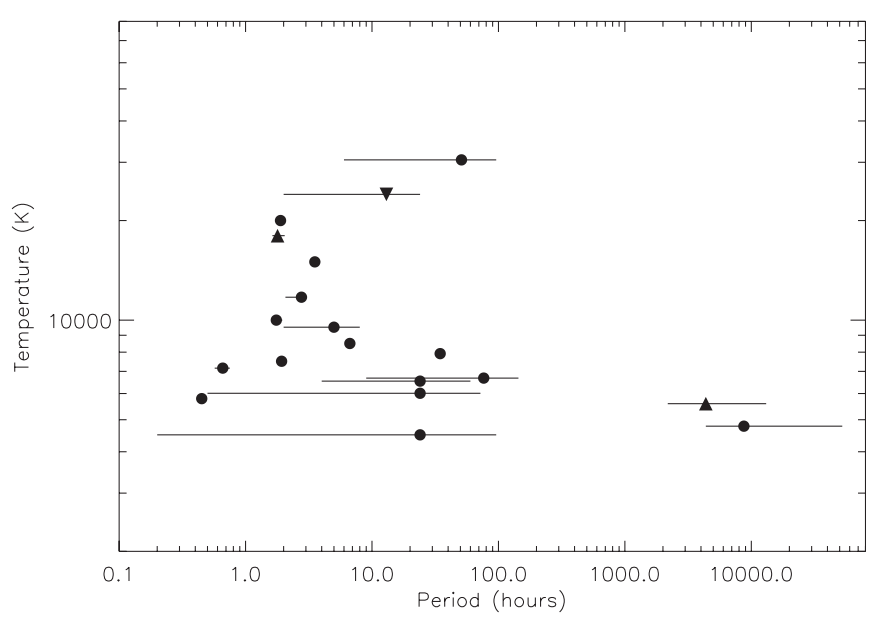

Figure 28. Plot of effective temperature vs. measured period for our variable white dwarf sample. Also included are WD1953-011 and GD356, from Brinkworth et al. (2004, 2005). The shape of the plotting symbols describes the white dwarf composition-DA white dwarfs are plotted as circles, DB white dwarfs as upright triangles, while targets that have been reported as both DA and DB are plotted as upside-down triangles. We find no correlation between effective temperature and spin period in our sample.

\subsection{Correlations between Rotation Period and Other White Dwarf Parameters}

We searched for correlations between the period of variability (which we interpret as the spin period, for reasons described earlier) and the field strength, mass, temperature, and age of the stars. For the sources without a well-defined period, we simply used the best estimate from the periodogram, with the error bars covering the possible range of periods over $3 \sigma$ (Figures 27-30). We find no correlation between spin period and any other tested parameter. Wickramasinghe \& Ferrario (2000) found a positive correlation between period and magnetic field strength at shorter periods, and Ferrario \& Wickramasinghe (2005) found a similar result when including the slow rotators, but we find no evidence for this in our data.

Interestingly, we have discovered variability in eight targets with both low temperatures $(T<12,000 \mathrm{~K})$ and low magnetic

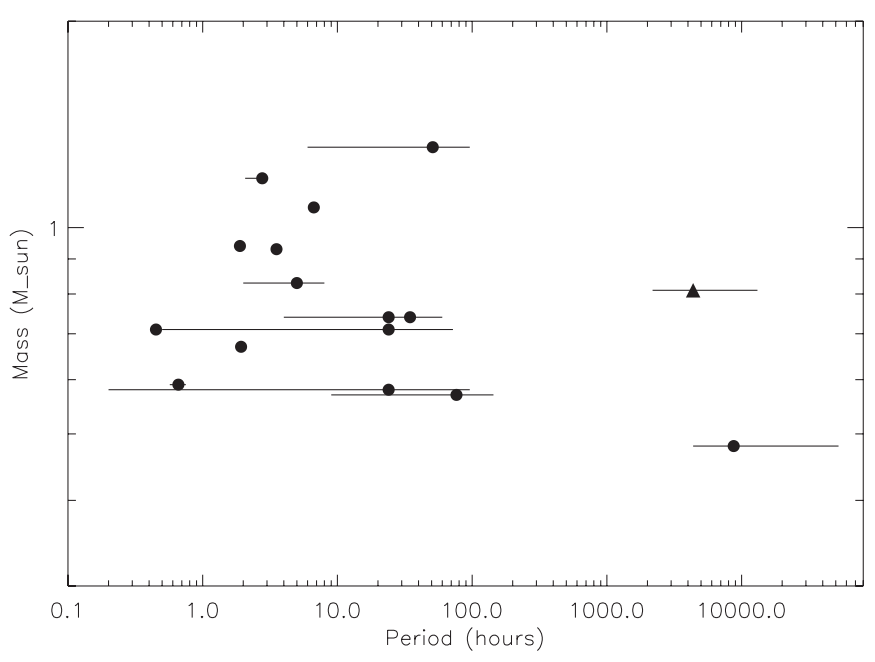

Figure 29. Plot of mass vs. measured period for our variable white dwarf sample. Also included are WD1953-011 and GD356, from Brinkworth et al. (2004, 2005). The shape of the plotting symbols describes the white dwarf composition-DA white dwarfs are plotted as circles, DB white dwarfs as upright triangles, while targets that have been reported as both DA and DB are plotted as upside-down triangles. We find no correlation between mass and spin period in our sample.

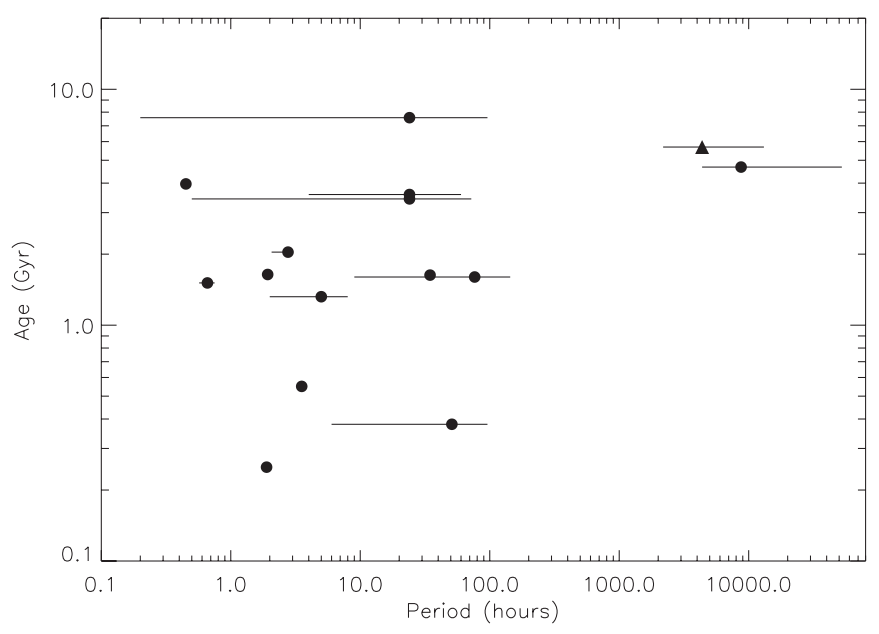

Figure 30. Plot of age vs. measured period for our variable white dwarf sample. Also included are WD1953-011 and GD356, from Brinkworth et al. (2004, 2005). The shape of the plotting symbols describes the white dwarf composition-DA white dwarfs are plotted as circles, DB white dwarfs as upright triangles, while targets that have been reported as both DA and DB are plotted as upside-down triangles. We find no correlation between white dwarf age and spin period in our sample.

field strengths $(B<20 \mathrm{MG})$. The most likely explanation for this is the presence of starspots on their surfaces, as seen in WD1953-011 (Brinkworth et al. 2005) and GD356 (Brinkworth et al. 2004). This is possibly due to the partially convective atmospheres of these low-temperature targets. Followup polarimetry of these eight targets could confirm the presence of starspots.

More surprisingly, we also find variability in two targets with low magnetic field strength, but high temperatures $(T>$ 20,000 K): LB8915 and PG1658+441. LB8915 is a DBA white dwarf, i.e., it has a helium dominated atmosphere with weak hydrogen lines in its optical spectrum. At $T_{\text {eff }} \sim 20,000 \mathrm{~K}$, it may still have a partially convective atmosphere, and so may still be able to form starspots despite its high temperature. Combined with data from Wesemael et al. (2001) showing polarimetric 
variability, it seems likely that the variability in LB8915 is due to magnetic field effects, most likely a starspot. PG1658+441, on the other hand, is a hydrogen-rich magnetic DA white dwarf with a field strength (3.5 MG) too low to cause magnetic dichroism and, at $T_{\text {eff }}=30,500 \mathrm{~K}$, a fully radiative atmosphere that cannot form starspots. Infrared observations at Two Micron All Sky Survey and Spitzer Space Telescope wavelengths find no IR excess indicative of a companion (Hansen et al. 2006), with an upper limit on an unresolved companion of 2 Jupiter masses (Farihi et al. 2008). The source of variability in PG1658 therefore remains a mystery.

\section{CONCLUSIONS}

Of 30 isolated magnetic white dwarfs, we find that 9 were untestable due to varying comparison stars. Of the remaining 21 , 14 show evidence for variability (67\%), and 7 show no evidence of variability and would benefit from further observations. The 9 discarded for having variable comparison stars would also benefit from repeat observations using a different field of view. Long-term follow-up of a number of the targets is currently underway.

We find no evidence for any correlation between spin period and any other white dwarf parameters, but note that few of our targets have well-defined periods. Our long-term follow-up should provide better constraints.

The authors would like to thank the referee for the careful helpful comments that greatly improved this paper.

Matt Burleigh acknowledges the support of a PPARC/STFC Advanced Fellowship during the course of this project. Tom Marsh acknowledges support from STFC during the course of this research.

The Jacobus Kapteyn Telescope is operated on the island of La Palma by the Isaac Newton Group in the Spanish Observatorio del Roque de los Muchachos of the Instituto de Astrofsica de Canarias. This research has made use of the SIMBAD database, operated at CDS, Strasbourg, France. This research has made use of NASA's Astrophysics Data System Bibliographic Services.

\section{REFERENCES}

Angel, J. R. P. 1978, ARA\&A, 16, 487

Angel, J. R. P., Carswell, R. F., Beaver, E. A., Harms, R., \& Strittmatter, P. A. 1974, ApJL, 194, L47

Barstow, M. A., Jordan, S., O'Donoghue, D., et al. 1995, MNRAS, 277, 971

Berdyugin, A. V., \& Pirola, V. 1999, A\&A, 352, 619

Berger, L., Koester, D., Napiwotzki, R., Reid, I. N., \& Zuckerman, B. 2005, A\&A, 444, 565

Bergeron, P., Leggett, S. K., \& Ruiz, M. T. 2001, ApJS, 133, 413

Bergeron, P., Ruiz, M. T., \& Leggett, S. K. 1997, ApJS, 108, 339

Brinkworth, C. S., Burleigh, M. R., Wynn, G. A., \& Marsh, T. R. 2004, MNRAS, 384, L33

Brinkworth, C. S., Marsh, T. R., Morales-Rueda, L., et al. 2005, MNRAS, 357,333

Bues, I., \& Pragal, M. 1989, LNP, 328, 329

Burleigh, M. R., Jordan, S., \& Schweizer, W. 1999, ApJL, 510, L37

Cohen, M. H., Putney, A., \& Goodrich, R. W. 1993, ApJL, 405, L67

Cumming, A., Marcy, G. W., \& Butler, R. P. 1999, ApJ, 526, 890

Dhillon, V. S., Marsh, T. R., Stevenson, M. J., et al. 2007, MNRAS, 378, 825

Dufour, P., Bergeron, P., \& Fontaine, G. 2005, ApJ, 627, 404

Dupuis, J., Vennes, S., \& Chayer, P. 2002, ApJ, 580, 1091

Euchner, F., Jordan, S., Beuermann, K., Reinsch, K., \& Gänsicke, B. T. 2006, A\&A, 451, 671

Euchner, F., Reinsch, K., Jordan, S., Beuermann, K., \& Gänsicke, B. T. 2005, A\&A, 442, 651

Farihi, J., Becklin, E. E., \& Zuckerman, B. 2008, ApJ, 681, 1470

Ferrario, L., Vennes, S., Wickramasinghe, D. T., Bailey, J., \& Christian, D. J. 1997, MNRAS, 292, 205
Ferrario, L., \& Wickramasinghe, D. T. 2005, MNRAS, 356, 615

Friedrich, S., Oestreicher, R., \& Schweizer, W. 1996, A\&A, 309, 227

Garcia-Berro, E., Loren-Aguilar, P., Aznar-Siguan, G., et al. 2012, ApJ, 749, 25

Glenn, J., Liebert, J., \& Schmidt, G. D. 1994, PASP, 106, 722

Green, R. F., \& Liebert, J. 1981, PASP, 93, 105

Greenstein, J. L. 1986, ApJ, 304, 334

Greenstein, J. L., \& McCarthy, J. K. 1985, ApJ, 289, 732

Greenstein, J. L., Oke, J. B., Richstone, D., Altena, W. F. V., \& Steppe, H. 1977, ApJL, 218, L21

Guseinov, O. H., Novruzova, H. I., \& Rustamov, Yu. S. 1983a, Ap\&SS, 97, 305 Guseinov, O. H., Novruzova, H. I., \& Rustamov, Yu. S. 1983b, Ap\&SS, 96, 1 Hansen, B. M. S., Kulkarni, S., \& Wiktorowicz, S. 2006, AJ, 131, 1106

Hermes, J. J., Montgomery, M. H., Winget, D. E., et al. 2012, ApJL, 750, L28

Holberg, J. B., Sion, E. M., Oswalt, T., et al. 2008, AJ, 135, 1225

Jordan, S., Aznar Cuadrado, R., Napiwotzki, R., Schmid, H. M., \& Solanki, S. K. 2007, A\&A, 462, 1097

Jordan, S., Schmelcher, P., \& Becken, W. 2001, A\&A, 376, 614

Karl, C. A., Napiwotzki, R., Heber, U., et al. 2005, A\&A, 434, 637

Kawaler, S. D. 2004, in IAU Symp. 215, Stellar Rotation, ed. A. Maeder \& P. Eenens (San Francisco, CA: ASP), 561

Kawka, A., \& Vennes, S. 2004, in IAU Symp. 224, The A-Star Puzzle, ed. J. Zverko, J. Ziznovsky, S. J. Adelman, \& W. W. Weiss (Cambridge: Cambridge Univ. Press), 879

Kawka, A., Vennes, S., Schmidt, G. D., Wickramasinghe, D. T., \& Koch, R. 2007, ApJ, 654, 499

Kepler, S. O., Giovannini, O., Wood, M. A., et al. 1995, ApJ, 447, 874

Kepler, S. O., Pelisoli, I., Jordan, S., et al. 2013, MNRAS, 429, 2934

King, A. R., Pringle, J. E., \& Wickramasinghe, D. T. 2001, MNRAS, 320, L45

Kleinman, S. J., Kepler, S. O., Koester, D., et al. 2013, ApJS, 204, 5

Koester, D., Dreizler, S., Weidermann, V., \& Allard, N. F. 1998, A\&A, 338, 612

Külebi, B., Jordan, S., Euchner, F., Gänsicke, B. T., \& Hirsch, H. 2009, A\&A, 506,1341

Liebert, J. 1976, PASP, 88, 490

Liebert, J., Bergeron, P., \& Holberg, J. B. 2003, AJ, 125, 348

Liebert, J., Bergeron, P., Schmidt, G. D., \& Saffer, R. A. 1993, ApJ, 418, 426

Liebert, J., Schmidt, G. D., Green, R. F., Stockman, H. S., \& McGraw, J. T. 1983, ApJ, 264, 262

Liebert, J., Schmidt, G. D., Sion, E. M., et al. 1985, PASP, 97, 158

Liebert, J., Smith, G. D., Lesser, M., et al. 1994, ApJ, 421, 733

Liebert, J., Wickramsinghe, D. T., Schmidt, G. D., et al. 2005, AJ, 129, 2376

Limoges, M.-M., \& Bergeron, P. 2010, ApJ, 714, 1037

Lomb, N. R. 1976, Ap\&SS, 39, 447

Maxted, P. F. L., Ferrario, L., Marsh, T. R., \& Wickramasinghe, D. T. 2000, MNRAS, 315, L41

Morales-Rueda, L., Maxted, P. F. L., Marsh, T. R., North, R. C., \& Heber, U. 2003, MNRAS, 338, 752

Moran, C., Marsh, T. R., \& Dhillon, V. S. 1998, MNRAS, 299, 218

Mukadam, A. S., Montgomery, M. H., Kim, A., et al. 2006, ApJ, 640, 956

Nelan, E. P. 2007, AJ, 134, 1934

Nordhaus, J., Wellons, S., Spiegel, D. S., Metzger, B. D., \& Blackman, E. G. 2011, PNAS, 108, 3135

Putney, A. 1995, ApJL, 451, L67

Putney, A. 1997, ApJS, 112, 527

Putney, A., \& Jordan, S. 1995, ApJ, 449, 863

Reimers, D., Jordan, S., Beckmann, V., Christlieb, N., \& Wisotzki, L. 1998, A\&A, 337, L13

Reimers, D., Jordan, S., Koehler, T., \& Wisotzki, L. 1994, A\&A, 285, 995

Reimers, D., Jordan, S., Koester, D., et al. 1996, A\&A, 311, 572

Scargle, J. D. 1982, ApJ, 263, 835

Schmidt, G. D., Allen, R. G., Smith, P. S., \& Liebert, J. 1996, ApJ, 463, 320

Schmidt, G. D., Bergeron, P., Liebert, J., \& Saffer, R. A. 1992a, ApJ, 394, 693

Schmidt, G. D., Harris, H. C., Leibert, J., et al. 2003, ApJ, 595, 1101

Schmidt, G. D., Liebert, J., \& Smith, P. S. 1998, ApJ, 116, 451

Schmidt, G. D., \& Norsworthy, J. E. 1991, ApJ, 366, 270

Schmidt, G. D., \& Smith, P. S. 1995, ApJ, 448, 305

Schmidt, G. D., Stockman, H. S., \& Smith, P. S. 1992b, ApJL, 398, L57

Schmidt, G. D., Vennes, S., Wickramasinghe, D. T., \& Ferrario, L. 2001, MNRAS, 328, 203

Schmidt, G. D., West, S. C., Liebert, J., Greeen, R. F., \& Stockman, H. S. 1986, ApJ, 309, 218

Sion, E. H., Holberg, J. B., Oswalt, T. D., McCook, G. P., \& Wasatonic, R. 2009, AJ, 138, 1681

Spruit, H. C. 1998, A\&A, 333, 603

Tout, C. A., Wickramasinghe, D. T., Liebert, J., Ferrario, L., \& Pringle, J. E. 2008, MNRAS, 387, 897

Valyavin, G., Antonyuk, K., Plachinda, S., et al. 2011, ApJ, 734, 17

Valyavin, G., Bagnulo, S., Monin, D., et al. 2005, A\&A, 439, 1099 
Vanlandingham, K. M., Schmidt, G. D., Eisenstein, D. J., et al. 2005, AJ, 130,734

Vennes, S., Ferrario, L., \& Wickramasinghe, D. T. 1999, MNRAS, 302, L49

Vennes, S., Schmidt, G. D., Ferrario, L., et al. 2003, ApJ, 593, 1040

Wesemael, F., Liebert, J., Schmidt, G. D., et al. 2001, ApJ, 554, 1118

Wickramasinghe, D. T., \& Cropper, M. 1988, MNRAS, 235, 1451
Wickramasinghe, D. T., \& Ferrario, L. 1988, ApJ, 327, 222

Wickramasinghe, D. T., \& Ferrario, L. 2000, PASP, 112, 873

Wickramasinghe, D. T., \& Ferrario, L. 2005, MNRAS, 356, 1576

Wickramasinghe, D. T., \& Martin, B. 1979, MNRAS, 188, 165

Wickramasinghe, D. T., Schmidt, G., Ferrario, L., \& Vennes, S. 2002, MNRAS, 332, 29

Winget, D. E., \& Kepler, S. O. 2008, ARA\&A, 46, 157 